\title{
Map Lattices Coupled by Collisions: Hitting Time Statistics and Collisions Per Lattice Unit
}

\author{
Wael Bahsoun and Fanni M. Sélley
}

\begin{abstract}
We study map lattices coupled by collision and show how perturbations of transfer operators associated with the spatially periodic approximation of the model can be used to extract information about collisions per lattice unit. More precisely, we study a map on a finite box of $L$ sites with periodic boundary conditions, coupled by collision. We derive, via a non-trivial first-order approximation for the leading eigenvalue of the rare event transfer operator, a formula for the first collision rate and a corresponding first hitting time law. For the former we show that the formula scales at the order of $L \cdot \varepsilon^{2}$, where $\varepsilon$ is the coupling strength, and for the latter, by tracking the $L$ dependency in our arguments, we show that the error in the law is of order $O\left(C(L) \frac{L \varepsilon^{2}}{\zeta(L)} \cdot\left|\ln \frac{L \varepsilon^{2}}{\zeta(L)}\right|\right)$, where $\zeta(L)$ is given in terms of the spectral gap of the rare event transfer operator, and $C(L)$ has an explicit expression. Finally, we derive an explicit formula for the first collision rate per lattice unit.
\end{abstract}

Mathematics Subject Classification. Primary 37A05, 37E05.

\section{Introduction}

Coupled map lattices are discrete-time dynamical systems modeling the interaction between microscopic units organized in a spatial structure given by a

The research of W. Bahsoun is supported by EPSRC Grant EP/V053493/1. The research of W. B. was partially carried out during a sabbatical supported by a Loughborough University Fellowship scheme. The research of F. M. Sélley was supported by the European Research Council (ERC) under the European Union's Horizon 2020 research and innovation programme (Grant Agreement No. 787304). The research of F. M. Sélley was partially carried out at Université de Paris and Sorbonne Université, CNRS, Laboratoire de Probabilités, Statistique et Modélisation, F-75013 Paris, France. 
lattice. The state of each unit, called a site, evolves according to the interplay between the local dynamics and the effect of the interaction with other sites. Such systems were introduced in the 80s (see [26] and references therein), and since then, they have seen a remarkable amount of research due to their paramount importance in applications and in studying non-equilibrium thermodynamics $[5,31]$. From a mathematical viewpoint, the main interest of coupled map lattices is that they provide natural examples of dynamical systems with an infinite-dimensional state space. Ergodic and statistical properties of coupled maps were studied extensively since the seminal work of [8]. Existence and uniqueness of the SRB measure and decay rate of correlations were the questions studied initially, typically in the weak interaction regime. The early results consider smooth expanding or hyperbolic local dynamics with coupling of similar regularity, while piecewise expanding site dynamics with more general coupling schemes (allowing to treat nearest neighbor coupling, for instance) were considered subsequently. See [10,22] for an extensive list of references. Considering the complete coupling regime, it is natural to expect phase transition-like phenomena. Such results were only proved in case of finite lattices with a small number of sites [25,33]; however, numerics suggest that the existence of multiple positive Lebesgue measure ergodic components can prevail for large system sizes [14]. Another line of current research studies coupled maps in more complex spatial structures. After the pioneering work of [27], more realistic setups were considered in the form of heterogeneous networks [29].

A natural way to understand a system on an infinite lattice is to consider its spatially periodic approximations, that is, coupled maps defined on finite boxes of sites with periodic boundary conditions. By understanding how various important dynamical quantities, such as entropy [12], escape rates in open systems [4], scale with the size of the system it is possible to meaningfully define the amount of "chaos" per lattice unit ${ }^{1}$ in the system [see [34] section 4.2 for a highlight about the importance of this concept in large systems]. A progress in studying the above quantities, entropy and escape rates, has been obtained in $[4,12]$ for one-dimensional lattices weakly coupled via a convolution operator.

In this work, we study coupled map lattices where the interaction takes place via rare but intense 'collisions' and the dynamics on each site is given by a piecewise uniformly expanding map of the interval. Our main goal is to quantify explicitly the amount of chaos per lattice unit in this model. The peculiarity of the model is that this kind of interaction gives rise to a discontinuous coupling function. Many of the technical challenges this poses were tackled in [23], where the existence of a unique Sinai-Ruelle-Bowen measure and exponential decay of correlations within a suitable class of measures was proved via a decoupling technique. $^{2}$ The motivation for this model can be understood through the intimate connections with similar models that are used by mathematicians

\footnotetext{
${ }^{1}$ See [11] for an introduction of this concept.

${ }^{2}$ See also $[21,22]$ where this technique was successfully applied for other types of coupling.
} 
and physicists in the study of heat transfer and the derivation of an associated Fourier's law $[3,15]$.

In this paper, we show how spectral techniques of transfer operators associated with the (finite-dimensional) periodic approximation of the model ${ }^{3}$ can be used to extract information about collisions per lattice unit. In particular, using this technique, a formula for the first collision rate with respect to Lebesgue measure, i.e., the fraction of orbits that see a first collision at each time step of the dynamics, ${ }^{4}$ and a corresponding hitting time distribution for the system of $L$ sites are derived. For the former we show that the formula scales at the order of $L \cdot \varepsilon^{2}$, where $\varepsilon$ is the coupling strength, and for the latter we show that the error in the law is of order $O\left(C(L) \frac{L \varepsilon^{2}}{\zeta(L)} \cdot\left|\ln \frac{L \varepsilon^{2}}{\zeta(L)}\right|\right)$, where $\zeta(L)$ is given in terms of the speed of mixing, and $C(L)$ is a particular function which is obtained by tracking the $L$ dependency in the abstract perturbation result of [24]. Moreover, we derive an explicit formula for the first collision rate per lattice unit. To the best of our knowledge explicit formulae of this type did not appear before this work in the mathematical literature. We believe that the transfer operator technique employed in this work can be applied to different types of coupled map lattices addressing a related problem, for instance, to derive a precise formula for the escape rate of the open system studied in $[4]$.

In Sect. 2 we introduce the coupled model, state the assumptions on the local dynamics, introduce the corresponding transfer operators and the function space on which the transfer operators act. We finish this section with the statement of our main results, Theorem 2.5 and Theorem 2.8. In Sect. 3 we provide examples to illustrate Theorem 2.5 ; in particular, we describe in certain low-dimensional cases the structure of the set of collision states, its limit set as the coupling strength goes to zero, and the formula of the 'derivative' for the dominant eigenvalue of a rare event transfer operator. Section 4 contains some technical lemmas, their proofs, and the proofs of Theorems 2.5 and 2.8. It also contains a remark discussing the infinite-dimensional limit of the collision rate and its per lattice unit counterpart.

\section{Setup}

Let $I$ denote the closed unit interval $[0,1]$. Let $X=I^{\Lambda}$, where $\Lambda=\mathbb{Z}^{d} /(N \mathbb{Z})^{d}$ for some integers $N, d \geq 1$. As an ease of notation, we are going to write $L=N^{d}$ from now on. We will refer to $\Lambda$ as the finite lattice of $L$ sites. We denote by $m_{L}$ the $L$-dimensional Lebesgue measure on $X$.

\footnotetext{
${ }^{3}$ In the infinite-dimensional system the space is not compact. Obtaining 'spectral data' for a transfer operator associated with the infinite-dimensional system is not obvious at all. On the other hand, this is well understood for a wide range of finite-dimensional systems similar to the coupled system arising from the periodic lattice.

${ }^{4}$ See Definition 2.2 for a precise statement.
} 


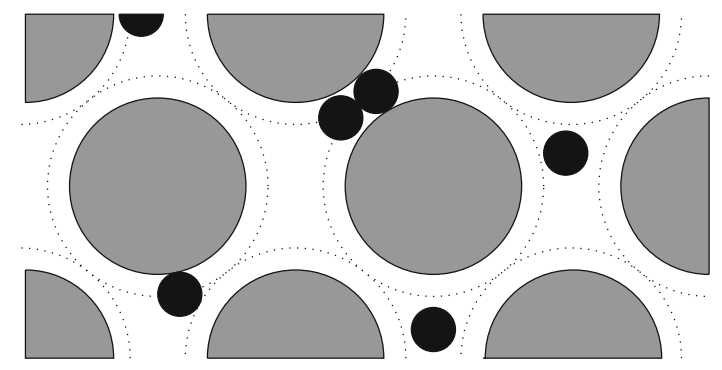

Figure 1. The Gaspard-Gilbert model [15]. Grey circles represent the obstacles, while the black circles are the moving billiard balls

Let $\tau: I \rightarrow I$ be the map describing the local dynamics at a single site. Define $T_{0}: X \rightarrow X$ as the product map $\left(T_{0}(x)\right)_{\mathbf{p}}=\tau\left(x_{\mathbf{p}}\right), \mathbf{p} \in \Lambda$, i.e., $T_{0}$ is the uncoupled dynamics on the lattice.

Let $V^{+}:=\left\{e_{i}\right\}$ be the standard basis of $\mathbb{R}^{d}$ and define $V:=V^{+} \cup-V^{+}$. For $\varepsilon>0$, let $\left\{A_{\varepsilon,-\mathbf{v}}\right\}_{\mathbf{v} \in V} \subset I$ be a set of disjoint open intervals, each of length $\varepsilon$. Denote by $\left(a_{\mathbf{v}}, a_{-\mathbf{v}}\right)$ the point that $A_{\varepsilon, \mathbf{v}} \times A_{\varepsilon,-\mathbf{v}}$ shrinks to as $\varepsilon \rightarrow 0$ and $S:=\left\{\left(a_{\mathbf{v}}, a_{-\mathbf{v}}\right)\right\}_{\mathbf{v} \in V^{+}}$be the set of all such points. Following [23], we consider the coupling ${ }^{5}$

$$
\left(\Phi_{\varepsilon}(x)\right)_{\mathbf{p}}= \begin{cases}x_{\mathbf{p}+\mathbf{v}} & \text { if } x_{\mathbf{p}} \in A_{\varepsilon, \mathbf{v}} \text { and } x_{\mathbf{p}+\mathbf{v}} \in A_{\varepsilon,-\mathbf{v}} \text { for some } \mathbf{v} \in V \\ x_{\mathbf{p}} & \text { otherwise }\end{cases}
$$

where $\mathbf{p}+\mathbf{v}$ is understood modulo $N$ coordinatewise. We define the coupled dynamics $T_{\varepsilon}: X \rightarrow X$ as the composition $T_{\varepsilon}:=\Phi_{\varepsilon} \circ T_{0}$.

We assume that $\tau: I \rightarrow I$ is piecewise monotone and $C^{1+\beta}$ on the intervals $I_{1}, \ldots, I_{k}$ with an extension of similar smoothness to the boundaries and $\left|\tau^{\prime}\right|>1$. We further assume that $\tau$ has full branches. It is well known that $\tau$ admits a unique absolutely continuous invariant measure $\mu_{\tau}$ whose density $h$ is bounded from below by a positive number on the whole interval $I[6]$, and it is furthermore well known to be (Hölder) continuous. Moreover, to study first-order approximations of collision rates, we assume that $\tau$ is continuous at $a_{\mathbf{v}}$ and $a_{-\mathbf{v}}$ for all $\mathbf{v} \in V$.

Remark 2.1. Our primary inspiration to study this this type of coupled map lattice was given by the paper [23]. In addition to this, an analogy to the billiard model of [15] provides further motivation: consider a periodic pattern of smooth scatterers, such as in Fig. 1. Among every four of them a cell is formed that contains a moving billiard ball. Thus, the cells have the structure of a two-dimensional lattice. The radius of the moving ball is large enough so that the ball is confined to its cell, but small enough so that it is able

\footnotetext{
${ }^{5}$ In fact the results of this paper apply to a more general form of coupling, regardless how $\Phi_{\varepsilon}$ is defined on $\left\{A_{\varepsilon,-\mathbf{v}}\right\}_{\mathbf{v} \in V}$, see Remark 2.3. We present the particular coupling (1) to link and apply our results to existing models in the literature, such as the one introduced in [23].
} 
to collide with the moving balls in each of the four neighboring cells. When no such interaction happens, the state of each ball (described by its position and velocity) evolves according to classical billiard dynamics. This can be understood as the local dynamics of the sites. When two moving balls collide, they exchange the components of their velocities that are parallel to the line through their centers at the moment of impact. This is similar in spirit to the coupling (1), where the state of two neighboring sites are interchanged if these states are in an appropriate zone with respect to each other, although in (1) the specific form of the collision is insignificant. As for the local dynamics, following [23] we consider the simpler setting of a piecewise uniformly expanding interval maps. The reason for this simplification is that the coupled system will be of similar regularity, but higher dimensional. Transfer operator techniques for multidimensional piecewise expanding maps are well understood $[16,28,32]$, in contrast to high-dimensional hyperbolic systems with singularites, ${ }^{6}$ which would have to be considered in case of the billiard model of [15].

In what follows we will build a theory to study the map on the finite lattice of $L$ sites.

\subsection{Transfer Operators, First Hitting Times and First Collision Rates}

Denote by $L_{\mathbb{R}^{L}}^{1}$ the space of measurable functions $f: \mathbb{R}^{L} \rightarrow \mathbb{R}$ for which

$$
\int_{\mathbb{R}^{L}}|f|<\infty .
$$

Let $L^{\infty}$ be the space of essentially bounded measurable functions $f: \mathbb{R}^{L} \rightarrow \mathbb{R}$. We will use the notation $\|f\|_{1}=\int|f|$ for $f \in L_{\mathbb{R}^{L}}^{1}$, and $\|f\|_{\infty}:=\operatorname{ess} \sup f$. Since $T_{0}$ is non-singular with respect to $m_{L}$, we can define the transfer operator associated with $T_{0}$, denoted by $P_{T_{0}}$, via the following duality: for $f \in L_{\mathbb{R}^{L}}^{1}$ and $g \in L^{\infty}$

$$
\int g \circ T_{0} \cdot f \mathrm{~d} m_{L}=\int g \cdot P_{T_{0}} f \mathrm{~d} m_{L} .
$$

Now, define $\mathcal{I}_{L}$ as the set of rectangles $I_{i_{1}} \times \cdots \times I_{i_{L}}, i_{1}, \ldots, i_{L} \in\{1, \ldots, k\}$ on which $T_{0}$ is $C^{1+\beta}$. Then, the transfer operator $P_{T_{0}}$ has the following a.e. pointwise representation:

$$
P_{T_{0}} f(x)=\sum_{J \in \mathcal{I}_{L}} \frac{f}{\left|\operatorname{det}\left(D T_{0}\right)\right|} \circ\left(T_{0} \mid J\right)^{-1}(x) 1_{T_{0}(J)}(x) .
$$

2.1.1. Rare Events and the Corresponding Transfer Operator. For $\mathbf{p} \in \Lambda$ let

$$
X_{\varepsilon, \mathbf{v}}(\mathbf{p})=\left\{x \in X: x_{\mathbf{p}} \notin A_{\varepsilon, \mathbf{v}} \text { or } x_{\mathbf{p}+\mathbf{v}} \notin A_{\varepsilon,-\mathbf{v}}\right\},
$$

and set

$$
X_{\varepsilon}^{0}:=\bigcap_{\mathbf{p} \in \Lambda} \bigcap_{\mathbf{v} \in V} X_{\varepsilon, \mathbf{v}}(\mathbf{p}),
$$

and

$$
H_{\varepsilon}:=X \backslash X_{\varepsilon}^{0}
$$

\footnotetext{
${ }^{6}$ See the introduction of the recent article [2] for an exposition and the current state of the art on transfer operator techniques for (piecewise) hyperbolic systems.
} 
Let

$$
X_{\varepsilon}^{n-1}=\cap_{i=0}^{n-1} T_{0}^{-i} X_{\varepsilon}^{0} .
$$

Notice that $X_{\varepsilon}^{n-1}$ is the set of points whose orbits did not result in any collision up to time $n-1$. For $n \geq 1$ and a measurable function $f$, let

$$
\hat{P}_{\varepsilon}^{n} f=P_{T_{0}}^{n}\left(1_{X_{\varepsilon}^{n-1}} f\right) .
$$

Following the terminology of [19], we are going to call $\hat{P}_{\varepsilon}$ the rare event transfer operator.

Note that in case of $\varepsilon=0$ we have $X_{0}^{n}=X$ for all $n \in \mathbb{N}$, and hence in this case the operator defined by (2) simply reduces to the transfer operator of $T_{0}$.

It is a natural question to study how long a typical trajectory of the coupled system can survive without experiencing a single collision.

Definition 2.2. Let $m$ be a probability measure supported on $X_{\varepsilon}^{0}$. Suppose that

$$
-\lim _{n \rightarrow \infty} \frac{1}{n} \ln m\left(X_{\varepsilon}^{n-1}\right):=\hat{r}
$$

exists. Then, the quantity in the above limit measures asymptotically, with respect to $m$, the fraction of orbits that see a first collision at each time step under the dynamics of $T_{\varepsilon}$. We call such a quantity the first collision rate under $T_{\varepsilon}$, with respect to $m$.

The quantity in Definition 2.2 captures the fact that the proportion of initial conditions that lead to no collision up to time $n$ shrinks exponentially with rate $\hat{r}$. The motivation behind this definition is to study how $\hat{r}$, when it exists, scales with the size of the system to meaningfully define the amount of "chaos" per lattice unit. This is achieved in Theorem 2.8 for the collision models we are interested in.

A natural subsequent question to the fist collision rate is to study how long it takes for a typical trajectory to experience its first collision. We define the first hitting time by

$$
t_{\varepsilon}(x)=\inf \left\{n \geq 0: T_{\varepsilon}^{n}(x) \in H_{\varepsilon}\right\}
$$

, i.e., $t_{\varepsilon}$ is the first time when we see a collision under the coupled map $T_{\varepsilon}$. Note that $t_{\varepsilon}$ can be equivalently defined by

$$
t_{\varepsilon}(x)=\inf \left\{n \geq 0: T_{0}^{n}(x) \in H_{\varepsilon}\right\} .
$$

Remark 2.3. The above defined quantities do not take into consideration how the coupling acts on states that involve a collision, since we study trajectories up until the first collision event occurs. More precisely, no coupling takes place on the trajectories we study, so in fact what we study can be viewed as the product map $T_{0}$ with a specific hole $H_{\varepsilon} \cdot{ }^{7}$

\footnotetext{
${ }^{7}$ For more information on systems with holes we refer the reader to [13] for a basic introduction and a review, to [30] for a recent account and to $[7,19]$ for their connections with hitting time statistics.
} 
The main goal of this work is to understand the first collision rates and hitting time statistics in map lattices coupled by collision as introduced by Keller and Liverani [23] and the generalizations of this collision model as described in footnote 1 of [23].

\subsection{Quasi-Hölder Spaces}

We will study the action of the transfer operators defined above on a suitable 8 Banach spaces. It turns out that for the problem at hand, the most suitable space is the one introduced in [32]. We start by recalling the function spaces introduced in [32].

Let $0<\alpha \leq 1$ and $\omega_{0}>0$ be parameters. Define the oscillation of $f$ on a Borel set $S \subset \overline{\mathbb{R}}^{L}$ as

$$
\operatorname{osc}(f, S)=\operatorname{essup}_{S} f-\operatorname{essinf}_{S} f .
$$

Let

$$
|f|_{\alpha}=\sup _{0<\omega \leq \omega_{0}} \omega^{-\alpha} \int_{\mathbb{R}^{L}} \operatorname{osc}\left(f, B_{\omega}(x)\right) \mathrm{d} x
$$

and

$$
V_{\alpha}=\left\{f \in L_{\mathbb{R}^{L}}^{1}:|f|_{\alpha}<\infty\right\} .
$$

Set $\|f\|_{\alpha}=|f|_{\alpha}+\|f\|_{1}$ for $f \in V_{\alpha}$. Then, by [ [32], Proposition 3.3] $\left(V_{\alpha},\|\cdot\|_{\alpha}\right)$ is a Banach space, and the intersection of the unit ball of $V_{\alpha}$ with the set of functions supported on $X$ is compact in $L_{\mathbb{R}^{L}}^{1}$. Moreover, by [ [32], Proposition 3.4] $V_{\alpha}$ is continuously injected in $L^{\infty}$; in particular, $\forall f \in V_{\alpha}$

$$
\|f\|_{\infty} \leq \frac{\max \left(1, \omega_{0}^{\alpha}\right)}{\gamma_{L} \omega_{0}^{L}}\|f\|_{\alpha},
$$

where $\gamma_{L}$ denotes the volume of the unit ball of $\mathbb{R}^{L}$. As this constant will be important to us on multiple occasions, we introduce the notation

$$
C\left(L, \omega_{0}\right)=\frac{\max \left(1, \omega_{0}^{\alpha}\right)}{\gamma_{L} \omega_{0}^{L}} .
$$

\subsection{Notation, Collision States and Their Limit Sets}

Now we introduce some notation that are needed in the statements of our main results. All the notation introduced in this subsection is illustrated through simple examples in Sect. 3. Recall that $H_{\varepsilon}=X \backslash X_{\varepsilon}^{0}$ is the set of points whose state results in a collision. Let

$$
H_{\varepsilon}^{\mathbf{p}, \mathbf{v}}=\left\{x \in X: x_{\mathbf{p}} \in A_{\varepsilon, \mathbf{v}}, x_{\mathbf{p}+\mathbf{v}} \in A_{\varepsilon,-\mathbf{v}}\right\}
$$

for $\mathbf{p} \in \Lambda, \mathbf{v} \in V^{+}$. This set denotes the set of points whose state results in a collision at site $\mathbf{p}$ in the direction $\mathbf{v}$. Then,

$$
H_{\varepsilon}=\cup_{\mathbf{p} \in \Lambda} \cup_{\mathbf{v} \in V^{+}} H_{\varepsilon}^{\mathbf{p}, \mathbf{v}} .
$$

\footnotetext{
${ }^{8}$ The choice of these spaces among others is almost necessary in this context. Indeed, to apply the perturbation results of [24] in higher dimensions, as in the case of the coupled systems we are dealing with, it is 'almost' necessary to use a Banach space that is contained in $L^{\infty}$.
} 
Note that this is a finite, but, in general, not a disjoint union as multiple collisions can happen at the same time.

Note that $H_{\varepsilon}^{\mathbf{p}, \mathbf{v}}$ are $L$-dimensional boxes with a base of area $\varepsilon^{2}$. Their limiting sets, as $\varepsilon \rightarrow 0$, will play a crucial role in the statement and proof of Theorem 2.5. Denote these limiting sets by $I_{\mathbf{p}, \mathbf{v}}=\lim _{\varepsilon \rightarrow 0} H_{\varepsilon}^{\mathbf{p}, \mathbf{v}}$. Explicitly they are given as

$$
I_{\mathbf{p}, \mathbf{v}}=\left\{x \in X: x_{\mathbf{p}}=a_{\mathbf{v}}, x_{\mathbf{p}+\mathbf{v}}=a_{-\mathbf{v}}\right\} .
$$

Remark 2.4. It is easy to see that $\lim _{\varepsilon \rightarrow 0} m_{L}\left(H_{\varepsilon}^{\mathbf{p}, \mathbf{v}}\right)=m_{L}\left(I_{\mathbf{p}, \mathbf{v}}\right)=0$. This implies that $m_{L}\left(H_{\varepsilon}\right) \rightarrow 0$ as $\varepsilon \rightarrow 0$.

Recall that for each $\mathbf{v} \in V^{+},\left(a_{\mathbf{v}}, a_{-\mathbf{v}}\right)$ denotes the point that $A_{\varepsilon, \mathbf{v}} \times A_{\varepsilon,-\mathbf{v}}$ shrinks to as $\varepsilon \rightarrow 0$ and let $S:=\left\{\left(a_{\mathbf{v}}, a_{-\mathbf{v}}\right)\right\}_{\mathbf{v} \in V^{+}}$be the set of all such points. Let $S^{\text {per }} \subset S$ be the set of periodic points in $S$ with respect to the map $T_{2,0}=\tau \times \tau$. For each $\left(a_{\mathbf{v}}, a_{-\mathbf{v}}\right) \in S^{\text {per }}$ define $k(\mathbf{v})$ as the smallest integer $k$ such that $T_{2,0}^{k+1}\left(a_{\mathbf{v}}, a_{-\mathbf{v}}\right) \in S$. Let $V_{k}^{+} \subset V^{+}$denote the set of directions for which $k(\mathbf{v})=k$ and $K=\{k(\mathbf{v})\}_{\mathbf{v} \in V^{+}}$. Note that the case when $S^{\text {per }} \neq \emptyset$ is interesting and important to study since, as the calculation below shows, the collision rate will be higher when the collision zones are centered around periodic points with higher minimal period. ${ }^{9}$

\subsection{Statements of the Main Results}

In the following theorem $L$ is fixed and we understand $o(1) \rightarrow 0$ as $\varepsilon \rightarrow 0$.

Theorem 2.5. Fix $L \in \mathbb{N}$. For $\varepsilon \geq 0$ sufficiently small, the operator $\hat{P}_{\varepsilon}: V_{\alpha} \rightarrow$ $V_{\alpha}$ admits a spectral gap. In particular $T_{0}$ admits an absolutely continuous invariant measure $\mu_{0}=\rho_{0} \cdot m_{L}$, with $\rho_{0}(x)=\Pi_{i=1}^{L} h\left(x_{i}\right)$. Moreover, for $\varepsilon>0$,

1. $\hat{P}_{\varepsilon}$ has an eigenfunction $\rho_{\varepsilon}>0, \int \rho_{\varepsilon} \mathrm{d} x=1$, corresponding to a simple dominant eigenvalue $\lambda_{\varepsilon} \in(0,1)$, with

$$
\lambda_{\varepsilon}=1-\mu_{0}\left(H_{\varepsilon}\right) \cdot \theta(1+o(1))
$$

where

$$
\mu_{0}\left(H_{\varepsilon}\right)=L \Xi_{\varepsilon}(1+o(1)) \sim \varepsilon^{2} L d
$$

such that

$$
\Xi_{\varepsilon}=\sum_{\mathbf{v} \in V^{+}} \int_{A_{\varepsilon, \mathbf{v}}} \int_{A_{\varepsilon,-\mathbf{v}}} h\left(x_{1}\right) h\left(x_{2}\right) \mathrm{d} x_{1} \mathrm{~d} x_{2},
$$

and $\theta$ is a constant. In particular,

- if $S^{\text {per }} \neq \emptyset$ then $^{10}$

$$
\theta=1-\frac{1}{\sum_{\mathbf{v} \in V^{+}} h\left(a_{\mathbf{v}}\right) h\left(a_{-\mathbf{v}}\right)} \sum_{k \in K} \sum_{\mathbf{v} \in V_{k}^{+}} \frac{h\left(a_{\mathbf{v}}\right) h\left(a_{-\mathbf{v}}\right)}{\left|\left(\tau^{k+1}\right)^{\prime}\left(a_{\mathbf{v}}\right)\left(\tau^{k+1}\right)^{\prime}\left(a_{-\mathbf{v}}\right)\right|} ;
$$

- otherwise $\theta=1$.

\footnotetext{
${ }^{9}$ This is inspired by the works of $[9,24]$ where this was observed in the study of escape rates in interval maps with holes.

${ }^{10}$ The formula of $\theta$ below is quite general. The reader is invited to go through the examples of Sect. 3 to see how it reduces significantly in specific settings.
} 
2. The first collision rate of $T_{\varepsilon}$ with respect to $m_{L}$

$$
\hat{r}_{L}:=-\lim _{n \rightarrow \infty} \frac{1}{n} \ln m_{L}\left(X_{\varepsilon}^{n-1}\right)
$$

exists and

$$
\hat{r}_{L}=\mu_{0}\left(H_{\varepsilon}\right) \cdot \theta(1+o(1)) .
$$

3. In addition, there exists $C>0, \xi_{\varepsilon}>0$, with $\lim _{\varepsilon \rightarrow 0} \xi_{\varepsilon}=\theta$, such that for all $t>0$

$$
\left|\mu_{0}\left\{t_{\varepsilon} \geq \frac{t}{\xi_{\varepsilon} \mu_{0}\left(H_{\varepsilon}\right)}\right\}-e^{-t}\right| \leq C(t \vee 1) e^{-t} C(L) \frac{L \mathrm{~d} \varepsilon^{2}}{\zeta(L)}\left|\ln \left(\frac{L \mathrm{~d} \varepsilon^{2}}{\zeta(L)}\right)\right|
$$

where $\zeta(L)$ is given in terms of the spectral gap of the rare event transfer operator (see (35)) and $C(L)=C\left(L, \bar{c}(\tau) L^{-1 / 2}\right)$ for $C(\cdot, \cdot)$ defined by (4).

Remark 2.6. The formula (6) simplifies considerably when $d=1$. In that case $V^{+}=\{1\}$ and $S=\left\{\left(a_{1}, a_{-1}\right)\right\}$. Supposing that $k$ is the smallest integer such that $T_{2,0}^{k+1}\left(a_{1}, a_{-1}\right)=\left(a_{1}, a_{-1}\right)$, we get

$$
\theta=1-\frac{1}{\left|\left(\tau^{k+1}\right)^{\prime}\left(a_{1}\right)\left(\tau^{k+1}\right)^{\prime}\left(a_{-1}\right)\right|},
$$

independently of the invariant density $h$ of $\tau$.

Remark 2.7. In the third item of Theorem 2.5 we provide a bound on the first hitting time law that depends on the time scale $t$. We follow the spectral approach of [19] (inspired by [1]) and compute the precise $L$ dependence of the error term. We note that the particular time-dependent bound we obtain improves as $t \rightarrow \infty$, so it is meaningful for large values $t$ : our goal with this statement is to give a bound on the decay of the tail of the hitting time distribution. We note that for small values of $t$, a uniform bound such as the one given in [18] could be more appropriate.

The proof of Theorem 2.5 follows closely the abstract argument of [24]. However, since we are interested in how dynamical quantities scale with the lattice size $L$, we carefully track the $L$ dependency of various quantities and error terms. Furthermore, we provide a precise formula for $\theta$ in this specific system (sometimes referred to in the literature as the extremal index), a result which is interesting on its own right. Tracking the $L$ dependency in the argument of [24] is useful in particular in identifying explicitly the constants $\zeta(L)$ and $C(L)$ in the third item of Theorem 2.5. Moreover, such understanding is essential to progress in the direction of computing the infinite-dimensional limit of the first collision rate per lattice unit. See Remark 4.5.

As the second item of Theorem 2.5 states, we have

$$
\hat{r}_{L}=\mu_{0}\left(H_{\varepsilon}\right) \cdot \theta(1+o(1)) .
$$

On the other hand the proof of Theorem 2.5 also shows that

$$
\mu_{0}\left(H_{\varepsilon}\right)=L \Xi_{\varepsilon}(1+o(1)) \sim \varepsilon^{2} L d
$$




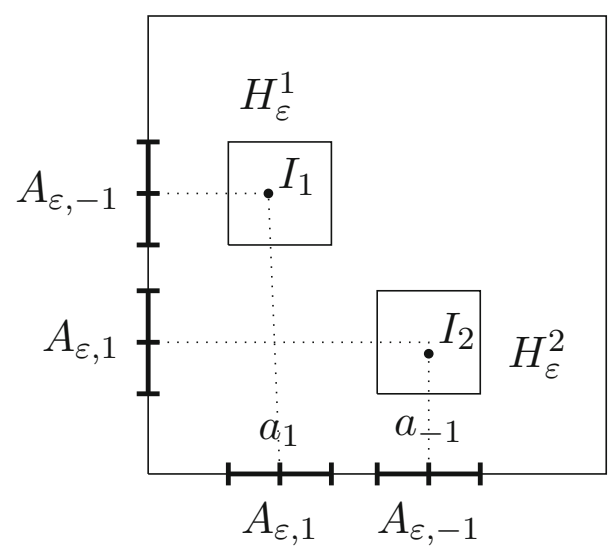

Figure 2. The state space when $L=2$ : the system with two interacting sites

and consequently $\hat{r}_{L}$, scales linearly in $L$. Thus, to obtain a first collision rate per lattice unit equation (8) suggests that a normalization by $L$ is needed. This is made precise in our second main theorem.

Theorem 2.8. For any $L \geq 2$, the first collision rate per lattice unit is given by

$$
\frac{1}{L} \hat{r}_{L}=\Xi_{\varepsilon} \cdot \theta(1+o(1))
$$

as $\varepsilon \rightarrow 0$, where $\Xi_{\varepsilon}$ and $\theta$ are as in Theorem 2.5.

Remark 2.9. Note that the little $o$ notation in Theorem 2.8 depends on $L$, so taking the limit $L \rightarrow \infty$ is not an obvious task. Again, see Remark 4.5.

\section{Examples}

In this section we provide examples to illustrate Theorem 2.5. In particular, we describe the structure of the set $H_{\varepsilon}$, its limit when $\varepsilon \rightarrow 0$ and the formula of $\theta$ (6) for some low-dimensional finite lattices.

\subsection{One-Dimensional Lattice}

Let $d=1$. We naturally identify the sites $\mathbf{p}_{1}, \ldots, \mathbf{p}_{L}$ with the numbers $1 \ldots, L$. Now $V^{+}=\{1\}$ and $S=\left(a_{1}, a_{-1}\right)$, so to simplify notation we will denote $H_{\varepsilon}^{\mathbf{p}_{i}, \mathbf{v}}$ as $H_{\varepsilon}^{i}$ and $I_{\mathbf{p}_{i}, \mathbf{v}}$ as $I_{i}$, as it causes no confusion.

Suppose $\left(a_{1}, a_{-1}\right)$ is a $k+1$ periodic point of $T_{2,0}$. We now study lattices of some small number of sites.

- $L=2: H_{\varepsilon}^{1}=A_{\varepsilon, 1} \times A_{\varepsilon,-1}$ and $H_{\varepsilon}^{2}=A_{\varepsilon,-1} \times A_{\varepsilon, 1}$ are two disjoint squares, while $I_{1}=\left(a_{1}, a_{-1}\right)$ and $I_{2}=\left(a_{-1}, a_{1}\right)$. Both squares represent the single possible interaction between the two sites. See Fig. 2 for an illustration. 


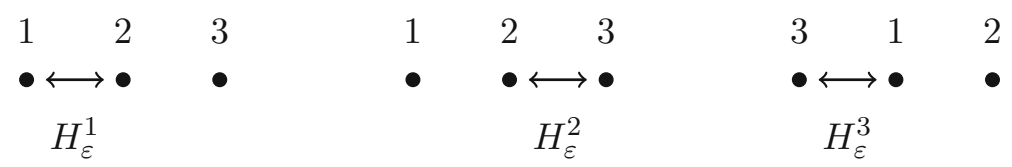

Figure 3. Interactions in case of $L=3$

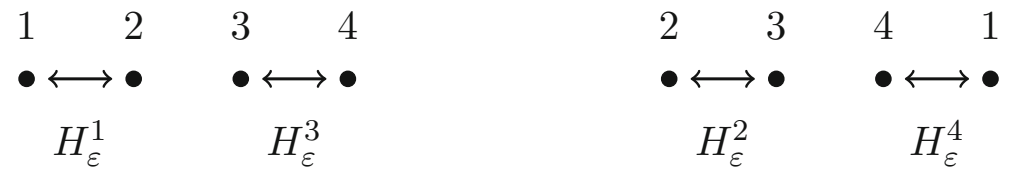

Figure 4. Possible simultaneous interactions when $d=1$, $L=4$

- $L=3: H_{\varepsilon}^{1}=A_{\varepsilon, 1} \times A_{\varepsilon,-1} \times I, H_{\varepsilon}^{2}=I \times A_{\varepsilon, 1} \times A_{\varepsilon,-1}$ and $H_{\varepsilon}^{3}$ $=A_{\varepsilon,-1} \times I \times A_{\varepsilon, 1}$, while their limits are the intervals $I_{1}=a_{1} \times a_{-1} \times I$, $I_{2}=I \times a_{1} \times a_{-1}$ and $I_{2}=a_{-1} \times I \times a_{1}$. Notice that since $A_{\varepsilon, 1}$ and $A_{\varepsilon,-1}$ are disjoint, the sets $H_{\varepsilon}^{i}, i=1,2,3$ are pairwise disjoint. This translates to the fact that we cannot have multiple interactions simultaneously. The set $H_{\varepsilon}^{1}$ corresponds to the interaction between sites 1 and $2, H_{\varepsilon}^{2}$ to 2 and 3 and $H_{\varepsilon}^{3}$ to 3 and 1. See Fig. 3 for an illustration.

- $L=4$ : now $H_{\varepsilon}^{1}=A_{\varepsilon, 1} \times A_{\varepsilon,-1} \times I \times I, H_{\varepsilon}^{2}=I \times A_{\varepsilon, 1} \times A_{\varepsilon,-1} \times I$, $H_{\varepsilon}^{3}=I \times I \times A_{\varepsilon, 1} \times A_{\varepsilon,-1}$ and $H_{\varepsilon}^{4}=A_{\varepsilon,-1} \times I \times I \times A_{\varepsilon, 1}$. Notice that $H_{\varepsilon}^{1}$ and $H_{\varepsilon}^{3}$ have a non-empty intersection, and so does $H_{\varepsilon}^{2}$ and $H_{\varepsilon}^{4}$ :

$$
\begin{aligned}
& H_{\varepsilon}^{1} \cap H_{\varepsilon}^{3}=A_{\varepsilon, 1} \times A_{\varepsilon,-1} \times A_{\varepsilon, 1} \times A_{\varepsilon,-1} \\
& H_{\varepsilon}^{2} \cap H_{\varepsilon}^{4}=A_{\varepsilon,-1} \times A_{\varepsilon, 1} \times A_{\varepsilon,-1} \times A_{\varepsilon, 1} .
\end{aligned}
$$

In terms of the coupled system, this means that sites 1 and 3 can simultaneously interact with their neighbors to the right, and so can sites 2 and 4. See Fig. 4.

According to Remark 2.6, we obtain in all three cases studied above

$$
\theta=1-\frac{1}{\left|\left(\tau^{k+1}\right)^{\prime}\left(a_{1}\right)\left(\tau^{k+1}\right)^{\prime}\left(a_{-1}\right)\right|},
$$

independently of $h$. This is easy to prove when the sets $H_{\varepsilon}^{i}$ are pairwise disjoint, as in the case of $L=2$ and $L=3$.

However, we have to be more careful when the intersections $H_{\varepsilon}^{i} \cap H_{\varepsilon}^{j}, i \neq j$ are not necessarily empty, although we eventually obtain the same formula. Explaining the reason for this through the example of $L=4$, the key fact the proof will depend on is that the limit sets $I_{1}=a_{1} \times a_{-1} \times I \times I, I_{2}$ $=I \times a_{1} \times a_{-1} \times I, I_{3}=I \times I \times a_{1} \times a_{-1}$ and $I_{4}=a_{-1} \times I \times I \times a_{1}$ are two-dimensional sets, but the intersections

$$
\begin{aligned}
& I_{1} \cap I_{3}=a_{1} \times a_{-1} \times a_{1} \times a_{-1} \\
& I_{2} \cap I_{4}=a_{-1} \times a_{1} \times a_{-1} \times a_{1}
\end{aligned}
$$




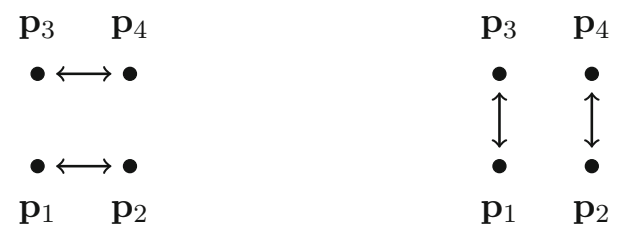

Figure 5. Possible simultaneous interactions when $d=2$, $L=4$. Left: corresponds to the intersection of $H_{\varepsilon}^{\mathbf{p}_{1}, \mathbf{h}}$ and $H_{\varepsilon}^{\mathbf{p}_{3}, \mathbf{h}}, H_{\varepsilon}^{\mathbf{p}_{2}, \mathbf{h}}$ and $H_{\varepsilon}^{\mathbf{p}_{4}, \mathbf{h}}, H_{\varepsilon}^{\mathbf{p}_{1}, \mathbf{h}}$ and $H_{\varepsilon}^{\mathbf{p}_{4}, \mathbf{h}}$ and $H_{\varepsilon}^{\mathbf{p}_{2}, \mathbf{h}}$ and $H_{\varepsilon}^{\mathbf{p}_{3}, \mathbf{h}}$; right: corresponds to the intersection of $H_{\varepsilon}^{\mathbf{p}_{1}, \mathbf{v}}$ and $H_{\varepsilon}^{\mathbf{p}_{2}, \mathbf{v}}, H_{\varepsilon}^{\mathbf{p}_{3}, \mathbf{v}}$ and $H_{\varepsilon}^{\mathbf{p}_{4}, \mathbf{v}}, H_{\varepsilon}^{\mathbf{p}_{1}, \mathbf{v}}$ and $H_{\varepsilon}^{\mathbf{p}_{4}, \mathbf{v}}$ and $H_{\varepsilon}^{\mathbf{p}_{2}, \mathbf{v}}$ and $H_{\varepsilon}^{\mathbf{p}_{3}, \mathbf{v}}$

are two points with zero two-dimensional Lebesgue measure, ${ }^{11}$ which will prove to be enough for them to be negligible in the computation of $\theta$.

\subsection{Two-Dimensional Lattice}

Let $d=2$ and $L=4$. Denote the sites by $\mathbf{p}_{1}=(0,0), \mathbf{p}_{2}=(1,0), \mathbf{p}_{3}=(0,1)$ and $\mathbf{p}_{4}=(1,1)$. Then, $V^{+}=\{\mathbf{v}, \mathbf{h}\}$, where $\mathbf{v}=(0,1)$ and $\mathbf{h}=(1,0)$. The set $S$ has two elements: $\left(a_{\mathbf{v}}, a_{-\mathbf{v}}\right)$ and $\left(a_{\mathbf{h}}, a_{-\mathbf{h}}\right)$. Suppose the minimal period of $\left(a_{\mathbf{v}}, a_{-\mathbf{v}}\right)$ is $k+1$, while the minimal period of $\left(a_{\mathbf{h}}, a_{-\mathbf{h}}\right)$ is $\ell+1$. If we study the sets $I_{\mathbf{p}_{i}, \mathbf{v}_{j}}$, we find that no three can intersect, but there are eight pairs that can intersect. Namely, $I_{\mathbf{p}_{1}, \mathbf{h}}$ and $I_{\mathbf{p}_{3}, \mathbf{h}} ; I_{\mathbf{p}_{2}, \mathbf{h}}$ and $I_{\mathbf{p}_{4}, \mathbf{h}} ; I_{\mathbf{p}_{1}, \mathbf{v}}$ and $I_{\mathbf{p}_{2}, \mathbf{v}}$; $I_{\mathbf{p}_{3}, \mathbf{v}}$ and $I_{\mathbf{p}_{4}, \mathbf{v}} ; I_{\mathbf{p}_{2}, \mathbf{h}}$ and $I_{\mathbf{p}_{3}, \mathbf{h}} ; I_{\mathbf{p}_{1}, \mathbf{h}}$ and $I_{\mathbf{p}_{4}, \mathbf{h}} ; I_{\mathbf{p}_{1}, \mathbf{v}}$ and $I_{\mathbf{p}_{4}, \mathbf{v}}$ and $I_{\mathbf{p}_{2}, \mathbf{v}}$ and $I_{\mathbf{p}_{3}, \mathbf{v}}$ (Fig. 5).

By Theorem 2.5 we obtain

$$
\begin{aligned}
\theta=1 & -\frac{1}{h\left(a_{\mathbf{v}}\right) h\left(a_{-\mathbf{v}}\right)+h\left(a_{\mathbf{h}}\right) h\left(a_{-\mathbf{h}}\right)} \cdot \frac{h\left(a_{\mathbf{v}}\right) h\left(a_{-\mathbf{v}}\right)}{\left|\left(\tau^{k+1}\right)^{\prime}\left(a_{\mathbf{v}}\right)\left(\tau^{k+1}\right)^{\prime}\left(a_{-\mathbf{v}}\right)\right|} \\
& +\frac{1}{h\left(a_{\mathbf{v}}\right) h\left(a_{-\mathbf{v}}\right)+h\left(a_{\mathbf{h}}\right) h\left(a_{-\mathbf{h}}\right)} \cdot \frac{h\left(a_{\mathbf{h}}\right) h\left(a_{-\mathbf{h}}\right)}{\left|\left(\tau^{\ell+1}\right)^{\prime}\left(a_{\mathbf{h}}\right)\left(\tau^{\ell+1}\right)^{\prime}\left(a_{-\mathbf{h}}\right)\right|} .
\end{aligned}
$$

So unless there is a close relation between the values at the points $a_{ \pm \mathbf{v}}$ and $a_{ \pm \mathbf{h}}$ of $h$ and the derivative of $\tau$, this expression depends non-trivially on the density of the site dynamics $\tau$.

\section{Proofs}

We first compute a formula for the measure of $H_{\varepsilon}$ with respect to the $T_{0^{-}}$ invariant measure $\mu_{0}=\Pi_{i=1}^{L} h \cdot m$.

\section{Lemma 4.1.}

$$
\mu_{0}\left(H_{\varepsilon}\right)=L \Xi_{\varepsilon}(1+o(1))
$$

\footnotetext{
${ }^{11}$ In the general case such intersections are not just points, but they will always be negligible relative to the appropriate Lebesgue measure.
} 
where

$$
\Xi_{\varepsilon}=\sum_{\mathbf{v} \in V^{+}} \int_{A_{\varepsilon, \mathbf{v}}} \int_{A_{\varepsilon,-\mathbf{v}}} h(x) h(y) \mathrm{d} x \mathrm{~d} y
$$

and $o(1) \rightarrow 0$ as $L \varepsilon^{2} \rightarrow 0$.

Proof. We start by making some observations that will considerably simplify the formula for $\mu_{0}\left(H_{\epsilon}\right)$. First notice that for each $\mathbf{p} \in \Lambda$ we must have $H_{\varepsilon}^{\mathbf{p}, \mathbf{v}_{i}} \cap$ $H_{\varepsilon}^{\mathbf{p}, \mathbf{v}_{j}}=\emptyset$ for $\mathbf{v}_{i} \neq \mathbf{v}_{j}$, as the state of site $\mathbf{p}$ cannot be simultaneously in $A_{\varepsilon, \mathbf{v}_{i}}$ and $A_{\varepsilon, \mathbf{v}_{j}}$. Notice also that at most $L / 2$ sets $H_{\varepsilon}^{\mathbf{p}_{i}, \mathbf{v}_{i}}$ can intersect: indeed, $x \in H_{\varepsilon}^{\mathbf{p}, \mathbf{v}}$ implies that $x_{\mathbf{p}} \in A_{\varepsilon, \mathbf{v}}$ for some $\mathbf{v} \in V^{ \pm}$and $x_{\mathbf{p}+\mathbf{v}}$ is in $A_{\varepsilon,-\mathbf{v}}$ where $-\mathbf{v} \in V^{\mp}$. This shows that $x \in H_{\varepsilon}^{\mathbf{p}, \mathbf{v}}$ implies that $x \notin H_{\varepsilon}^{\mathbf{p}+\mathbf{v}, \mathbf{v}^{\prime}}$ for any $\mathbf{v} \in V^{ \pm}$. So we can have $x$ in at most $L / 2$ sets $H_{\varepsilon}^{\mathbf{p}, \mathbf{v}}$ simultaneously.

Let $\Lambda^{k}=\left\{\left(\mathbf{p}_{1}, \ldots, \mathbf{p}_{k}\right): \mathbf{p}_{i} \in \Lambda, i=1, \ldots, k\right\}$ and $\left(V^{+}\right)^{k}=\left\{\left(\mathbf{v}_{1}, \ldots, \mathbf{v}_{k}\right)\right.$ : $\left.\mathbf{v}_{i} \in V^{+}, i=1, \ldots, k\right\}$. Fix an ordering on the elements of $\Lambda$ and denote it by $<$. Then, according to de Moivre's inclusion-exclusion principle and using the above observations we can write

$$
\begin{aligned}
& \mu_{0}\left(H_{\varepsilon}\right)=\sum_{\mathbf{p} \in \Lambda} \sum_{\mathbf{v} \in V^{+}} \mu_{0}\left(H_{\varepsilon}^{\mathbf{p}, \mathbf{v}}\right)-\sum_{\substack{\mathbf{p}_{1}, \mathbf{p}_{2} \in \Lambda \\
\mathbf{p}_{1}<\mathbf{p}_{2}}} \sum_{\mathbf{v}_{1}, \mathbf{v}_{2} \in V^{+}} \mu_{0}\left(H_{\varepsilon}^{\mathbf{p}_{1}, \mathbf{v}_{1}} \cap H_{\varepsilon}^{\mathbf{p}_{2}, \mathbf{v}_{2}}\right) \\
& +\sum_{\substack{\mathbf{p}_{1}, \mathbf{p}_{2}, \mathbf{p}_{3} \in \Lambda \\
\mathbf{p}_{1}<\mathbf{p}_{2}<\mathbf{p}_{3}}} \sum_{\mathbf{v}_{1}, \mathbf{v}_{2}, \mathbf{v}_{3} \in V^{+}} \mu_{0}\left(H_{\varepsilon}^{\mathbf{p}_{1}, \mathbf{v}_{1}} \cap H_{\varepsilon}^{\mathbf{p}_{2}, \mathbf{v}_{2}} \cap H_{\varepsilon}^{\mathbf{p}_{3}, \mathbf{v}_{3}}\right)-\ldots \\
& =\sum_{k=1}^{\lfloor L / 2\rfloor}(-1)^{k+1} \sum_{\substack{\left\{\mathbf{p}_{i}\right\}_{i=1}^{k} \in \Lambda^{k} \\
\mathbf{p}_{i}<\mathbf{p}_{i+1}}} \sum_{\left\{\mathbf{v}_{i}\right\}_{i=1}^{k} \in\left(V^{+}\right)^{k}} \mu_{0}\left(\bigcap_{i=1}^{k} H_{\varepsilon}^{\mathbf{p}_{i}, \mathbf{v}_{i}}\right) .
\end{aligned}
$$

Note that some intersections might be empty, but selecting the non-empty ones would make the formula unnecessarily complicated and would provide little benefit at this point. We also note here that the principle applied to a finite measure of density $F$ with respect to the Lebesgue measure implies that

$$
\int_{H_{\varepsilon}} F(x) \mathrm{d} x=\sum_{\ell=1}^{\lfloor L / 2\rfloor}(-1)^{\ell+1} \sum_{\substack{\left\{\mathbf{p}_{i}\right\}_{i=1}^{\ell} \in \Lambda^{\ell} \\ \mathbf{p}_{i}<\mathbf{p}_{i+1}}} \sum_{\left\{\mathbf{v}_{i}\right\}_{i=1}^{\ell} \in\left(V^{+}\right)^{\ell}} \int_{\cap_{i=1}^{\ell} H_{\varepsilon}^{\mathbf{p}_{i}, \mathbf{v}_{i}}} F(x) \mathrm{d} x .
$$

For $k=1$ we get

$$
\sum_{\mathbf{p} \in \Lambda} \sum_{\mathbf{v} \in V^{+}} \mu_{0}\left(H_{\varepsilon}^{\mathbf{p}, \mathbf{v}}\right)=L \sum_{\mathbf{v} \in V^{+}} \int_{A_{\varepsilon, \mathbf{v}}} \int_{A_{\varepsilon,-\mathbf{v}}} h\left(x_{1}\right) h\left(x_{2}\right) \mathrm{d} x_{1} \mathrm{~d} x_{2}=L \Xi_{\varepsilon} .
$$

where

$$
\Xi_{\varepsilon}=\sum_{\mathbf{v} \in V^{+}} \int_{A_{\varepsilon, \mathbf{v}}} \int_{A_{\varepsilon,-\mathbf{v}}} h\left(x_{1}\right) h\left(x_{2}\right) \mathrm{d} x_{1} \mathrm{~d} x_{2} .
$$

Now let $k>1$. We remind the reader that we denoted the density of the site dynamics $\tau$ by $h$. Since $h$ is of bounded variation, there exists $\bar{C}>0$ such that 
$h \leq \bar{C}$. If the intersection $\cap_{i=1}^{k} H_{\varepsilon}^{\mathbf{p}_{i}, \mathbf{v}_{i}}$ is non-empty we can write

$$
\begin{aligned}
\mu_{0} & \left(\bigcap_{i=1}^{k} H_{\varepsilon}^{\mathbf{p}_{i}, \mathbf{v}_{i}}\right) \\
& =\int_{A_{\varepsilon, \mathbf{v}_{1}}} \int_{A_{\varepsilon,-\mathbf{v}_{1}}} \ldots \int_{A_{\varepsilon, \mathbf{v}_{k}}} \int_{A_{\varepsilon,-\mathbf{v}_{k}}} \prod_{i=1}^{2 k} h\left(x_{i}\right) \mathrm{d} x_{1} \ldots \mathrm{d} x_{2 k} \\
& =\mu_{0}\left(H_{\varepsilon}^{\mathbf{p}_{1}, \mathbf{v}_{1}}\right) \int_{A_{\varepsilon, \mathbf{v}_{2}}} \int_{A_{\varepsilon,-\mathbf{v}_{2}}} \ldots \int_{A_{\varepsilon, \mathbf{v}_{k}}} \int_{A_{\varepsilon,-\mathbf{v}_{k}}} \prod_{i=3}^{2 k} h\left(x_{i}\right) \mathrm{d} x_{3} \ldots \mathrm{d} x_{2 k}
\end{aligned}
$$

which can be bounded by

$$
\mu_{0}\left(\bigcap_{i=1}^{k} H_{\varepsilon}^{\mathbf{p}_{i}, \mathbf{v}_{i}}\right) \leq \bar{C}^{2(k-1)} \varepsilon^{2(k-1)} \mu_{0}\left(H_{\varepsilon}^{\mathbf{p}_{1}, \mathbf{v}_{1}}\right)
$$

Now first notice that

$$
\mu_{0}\left(H_{\varepsilon}\right)=\mu_{0}\left(\bigcup_{\mathbf{p} \in \Lambda} \bigcup_{\mathbf{v} \in V^{+}} H_{\varepsilon}^{\mathbf{p}, \mathbf{v}}\right) \leq \sum_{\mathbf{p} \in \Lambda} \sum_{\mathbf{v} \in V^{+}} \mu_{0}\left(H_{\varepsilon}^{\mathbf{p}, \mathbf{v}}\right)=L \Xi_{\varepsilon}
$$

by (11).

For the lower bound we use (11) for $k=1$ and (12) for each $k>1$ :

$$
\begin{aligned}
& \sum_{k=1}^{\lfloor L / 2\rfloor}(-1)^{k+1} \sum_{\substack{\left\{\mathbf{p}_{i}\right\}_{i=1}^{k} \in \Lambda^{k} \\
\mathbf{p}_{i}<\mathbf{p}_{i+1}}} \sum_{\left\{\mathbf{v}_{i}\right\}_{i=1}^{k} \in\left(V^{+}\right)^{k}} \mu_{0}\left(\bigcap_{i=1}^{k} H_{\varepsilon}^{\mathbf{p}_{i}, \mathbf{v}_{i}}\right) \\
& =L \Xi_{\varepsilon}+\sum_{k=2}^{\lfloor L / 2\rfloor}(-1)^{k+1} \sum_{\substack{\left\{\mathbf{p}_{i}\right\}_{i=1}^{k} \in \Lambda^{k} \\
\mathbf{p}_{i}<\mathbf{p}_{j}}} \sum_{\left\{\mathbf{v}_{i}\right\}_{i=1}^{k} \in\left(V^{+}\right)^{k}} \mu_{0}\left(\bigcap_{i=1}^{k} H_{\varepsilon}^{\mathbf{p}_{i}, \mathbf{v}_{i}}\right) \\
& \geq L \Xi_{\varepsilon}-\left(\sum_{k=2}^{\lfloor L / 2\rfloor} \sum_{\substack{\left\{\mathbf{p}_{i}\right\}_{i=1}^{k} \in \Lambda^{k} \\
\mathbf{p}_{i}<\mathbf{p}_{i+1}}} \sum_{\left\{\mathbf{v}_{i}\right\}_{i=1}^{k} \in\left(V^{+}\right)^{k}} \mu_{0}\left(\bigcap_{i=1}^{k} H_{\varepsilon}^{\mathbf{p}_{i}, \mathbf{v}_{i}}\right)\right) \\
& \geq L \Xi_{\varepsilon}-\sum_{k=2}^{\lfloor L / 2\rfloor} \sum_{\substack{\left\{\mathbf{p}_{i}\right\}_{i=1}^{k} \in \Lambda^{k} \\
\mathbf{p}_{i}<\mathbf{p}_{i+1}}} \sum_{\left\{\mathbf{v}_{i}\right\}_{i=1}^{k} \in\left(V^{+}\right)^{k}} \bar{C}^{2(k-1)} \varepsilon^{2(k-1)} \mu_{0}\left(H_{\varepsilon}^{\mathbf{p}_{1}, \mathbf{v}_{1}}\right) \\
& =L \Xi_{\varepsilon}-\sum_{k=2}^{\lfloor L / 2\rfloor}\left(\begin{array}{c}
L \\
k-1
\end{array}\right) d^{k-1} \sum_{\mathbf{p}_{1} \in \Lambda} \sum_{\mathbf{v}_{1} \in V^{+}} \bar{C}^{2(k-1)} \varepsilon^{2(k-1)} \mu_{0}\left(H_{\varepsilon}^{\mathbf{p}_{1}, \mathbf{v}_{1}}\right) \\
& =L \Xi_{\varepsilon}-\left(\sum_{\mathbf{p}_{1} \in \Lambda} \sum_{\mathbf{v}_{1} \in V^{+}} \mu_{0}\left(H_{\varepsilon}^{\mathbf{p}_{1}, \mathbf{v}_{1}}\right)\right) \sum_{k=2}^{\lfloor L / 2\rfloor}\left(\begin{array}{c}
L \\
k-1
\end{array}\right) d^{k-1} \bar{C}^{2(k-1)} \varepsilon^{2(k-1)},
\end{aligned}
$$


which can be written as

$$
\mu_{0}\left(H_{\varepsilon}\right) \geq L \Xi_{\varepsilon}\left(1-\sum_{k=2}^{\lfloor L / 2\rfloor}\left(\begin{array}{c}
L \\
k-1
\end{array}\right) d^{k-1} \bar{C}^{2(k-1)} \varepsilon^{2(k-1)}\right) .
$$

The first term in the sum $\sum_{k=2}^{\lfloor L / 2\rfloor}\left(\begin{array}{c}L \\ k-1\end{array}\right) d^{k-1} \bar{C}^{2(k-1)} \varepsilon^{2(k-1)}$ is $L d \bar{C}^{2} \varepsilon^{2}$. This is the leading term assuming $L \varepsilon^{2}$ is sufficiently small, so

$$
\mu_{0}\left(H_{\varepsilon}\right) \geq L \Xi_{\varepsilon}\left(1-C L \mathrm{~d} \varepsilon^{2}\right)=L \Xi_{\varepsilon}(1+o(1)) .
$$

We now turn to the study of the transfer operators $\hat{P}_{\varepsilon}$. Observe that $T_{0}=\tau \times \cdots \times \tau$ satisfies conditions (PE1)-(PE4) of [32] for any $\alpha \leq \beta$. Indeed, the sets $U_{i}$ are given by the rectangles $I_{\underline{i}}=I_{i_{1}} \times \cdots \times I_{i_{L}}$ where $\underline{i}=\left(i_{1}, \ldots, i_{L}\right) \in$ $\{1, \ldots, k\}^{L}$. We further observe that since $\tau$ has full branches, that is, $\tau\left(I_{j}\right)=I$ for each $j=1, \ldots, k$, we have $T_{0}\left(I_{\underline{i}}\right)=I^{L}$ for all $\underline{i} \in\{1, \ldots, k\}^{L}$. We are going to use the notation $T_{0, \underline{i}}^{-1}=\left(\left.T_{0}\right|_{I_{\underline{i}}}\right)^{-1}$ for the inverse branches, defined on $I^{L}$. On the sets $I_{\underline{i}}$, the map $T_{0}$ is $C^{1+\beta}$ and can be extended to the boundary as a function of the same smoothness. This means that the first requirement of (PE2) about the piecewise smoothness of $T_{0}$ is satisfied, while the second requirement asking that for all $\underline{i}, \omega \leq \omega_{0}, z \in I^{L}$ and $x, y \in B_{\omega}(z) \cap I^{L}$

$$
\left|\operatorname{det} D_{x} T_{0, \underline{i}}^{-1}-\operatorname{det} D_{y} T_{0, \underline{i}}^{-1}\right| \leq c_{L}\left|\operatorname{det} D_{z} T_{0, \underline{i}}^{-1}\right| \omega^{\alpha},
$$

also holds, and we can choose $c_{L}=c(\tau) \sqrt{L}$. Finally, (PE4) requiring that for all $x, y \in I^{L}$

holds with $s:=\frac{1}{\min \left|\tau^{\prime}\right|}$.

$$
d\left(T_{0, \underline{i}}^{-1}(x), T_{0, \underline{i}}^{-1}(y)\right) \leq s d(x, y)
$$

Lemma 4.2. Let $n \in \mathbb{N}$ and $\varepsilon \geq 0$. There exist constants $\sigma \in(0,1), D\left(L, \varepsilon, \omega_{0}\right)$ $>0$ such that

$$
\begin{aligned}
& \left\|\hat{P}_{\varepsilon}^{n} f\right\|_{1} \leq\|f\|_{1} \\
& \left\|\hat{P}_{\varepsilon}^{n} f\right\|_{\alpha} \leq \sigma^{n}\|f\|_{\alpha}+D\left(L, \varepsilon, \omega_{0}\right)\|f\|_{1} .
\end{aligned}
$$

Proof. Since

$$
\left\|\hat{P}_{\varepsilon} f\right\|_{1}=\int_{X}\left|P_{T_{0}}\left(1_{X_{\varepsilon}^{0}} f\right)\right| \leq \int_{X}\left|1_{X_{\varepsilon}^{0}} f\right| \leq \int_{X}|f|,
$$

we have $\left\|\hat{P}_{\varepsilon}^{n} f\right\|_{1} \leq\|f\|_{1}$ for all $n \in \mathbb{N}$ and $\varepsilon \geq 0$.

To prove (16) we show the following:

$$
\begin{aligned}
\left\|P_{T_{0}} f\right\|_{\alpha} & \leq \sigma\|f\|_{\alpha}+K\|f\|_{1} ; \\
\left\|1_{X_{\varepsilon}^{0}} f\right\|_{\alpha} & \leq\|f\|_{\alpha}+8 L d \varepsilon \omega_{0}^{1-\alpha}\|f\|_{1},
\end{aligned}
$$

Writing $B\left(L, \varepsilon, \omega_{0}\right)=\sigma \cdot 8 L d \varepsilon \omega_{0}^{1-\alpha}+K$, the inequalities (17) and (18) imply that

$$
\left\|\hat{P}_{\varepsilon} f\right\|_{\alpha} \leq \sigma\|f\|_{\alpha}+B\left(L, \varepsilon, \omega_{0}\right)\|f\|_{1},
$$

which proves $(16)$ by iteration with $D\left(L, \varepsilon, \omega_{0}\right)=B\left(L, \varepsilon, \omega_{0}\right) \sum_{k=0}^{\infty} \sigma^{k}$. 
We first prove (17), arguing similarly as in Lemma 4.1 of [32], but in a simplified setting since $T_{0}$ is piecewise onto. ${ }^{12}$

$$
\begin{aligned}
\operatorname{osc}\left(P_{T_{0}} f, B_{\omega}(x)\right) & \leq \sum_{\underline{i}} \operatorname{osc}\left(\left(\frac{f}{\left|\operatorname{det} D T_{0}\right|}\right) \circ T_{0, \underline{i}}^{-1} \cdot \mathbf{1}_{T_{0}\left(I_{\underline{i}}\right)}, B_{\omega}(x)\right) \\
& =\sum_{\underline{i}} \operatorname{osc}\left(\left(\frac{f}{\left|\operatorname{det} D T_{0}\right|}\right) \circ T_{0, \underline{i}}^{-1}, B_{\omega}(x)\right) \\
& \leq \sum_{\underline{i}} \operatorname{osc}\left(\frac{f}{\left|\operatorname{det} D T_{0}\right|}, I_{\underline{i}} \cap T_{0}^{-1} B_{\omega}(x)\right) \\
& \leq \sum_{\underline{i}} \operatorname{osc}\left(\frac{f}{\left|\operatorname{det} D T_{0}\right|}, I_{\underline{i}} \cap B_{s \omega}\left(y_{\underline{i}}\right)\right)
\end{aligned}
$$

setting $y_{\underline{i}}=T_{0, \underline{i}}^{-1}(x)$ and remembering that $s$ was defined as the reciprocal of the minimum expansion of $\tau$ (and consequently $T_{0}$ ). It is easy to prove that for $g_{1}, g_{2} \in L^{\infty}, g_{2}>0$ and some $S$ Borel set

$$
\operatorname{osc}\left(g_{1} g_{2}, S\right) \leq \operatorname{osc}\left(g_{1}, S\right) \operatorname{ess}_{\sup _{S}} g_{2}+\operatorname{osc}\left(g_{2}, S\right) \operatorname{essinf} \operatorname{in}_{S}\left|g_{1}\right|
$$

holds. (The reader is invited to check Proposition 3.2 (iii) in [32].) This implies that

$$
\begin{aligned}
\operatorname{osc}\left(P_{T_{0}} f, B_{\omega}(x)\right) \leq & \sum_{\underline{i}} \operatorname{osc}\left(f, B_{s \omega}\left(y_{\underline{i}}\right)\right) \operatorname{ess}_{\sup _{\underline{\underline{i}} \cap} \cap B_{s \omega}\left(y_{\underline{i}}\right)} \frac{1}{\left|\operatorname{det} D T_{0}\right|} \\
& +\operatorname{osc}\left(\frac{1}{\left|\operatorname{det} D T_{0}\right|}, I_{\underline{i}} \cap B_{s \omega}\left(y_{\underline{i}}\right)\right) \operatorname{essinf}_{B_{s \omega}\left(y_{\underline{i}}\right)}|f| \\
\leq & \sum_{\underline{i}}\left(1+c_{L} s^{\alpha} \omega^{\alpha}\right) \operatorname{osc}\left(f, B_{s \omega}\left(y_{\underline{i}}\right)\right) \frac{1}{\left|\operatorname{det} D T_{0}\right|}\left(y_{\underline{i}}\right) \\
& +c_{L} s^{\alpha} \omega^{\alpha} \frac{1}{\left|\operatorname{det} D T_{0}\right|}\left(y_{\underline{i}}\right)|f|\left(y_{\underline{i}}\right) \\
\leq & \left(1+c_{L} s^{\alpha} \omega^{\alpha}\right) P_{T_{0}}\left(\operatorname{osc}\left(f, B_{s \omega}(x)\right)\right)+c_{L} s^{\alpha} \omega^{\alpha} P_{T_{0}}|f|(x),
\end{aligned}
$$

where $c_{L}$ and $s$ are given by (13) and (14), respectively. Integrating this on $\mathbb{R}^{L}$ we get

$$
\int_{\mathbb{R}^{L}} \operatorname{osc}\left(P_{T_{0}} f, B_{\omega}(\cdot)\right) \leq\left(1+c_{L} s^{\alpha} \omega^{\alpha}\right) \int_{\mathbb{R}^{L}} \operatorname{osc}\left(f, B_{s \omega}(\cdot)\right)+c_{L} s^{\alpha} \omega^{\alpha} \int_{\mathbb{R}^{L}}|f|,
$$

giving

$$
\left|P_{T_{0}} f\right|_{\alpha} \leq\left(1+c_{L} s^{\alpha} \omega_{0}^{\alpha}\right) s^{\alpha}|f|_{\alpha}+c_{L} s^{\alpha}\|f\|_{1}
$$

\footnotetext{
${ }^{12}$ In fact we include a detailed proof of (17) instead of using the general statement of Lemma 4.1 of [32] two reasons. First to track the $L$ dependency in all the constants. Second to show how assumptions on $\tau$ make it possible to avoid competition between the $L$ dependent complexity (which appears in the general argument of [32], see (PE5) and Lemma 2.1 of [32]) and the expansion of $\tau$. Indeed, had we used the general statement of Lemma 4.1 of [32], we would have the following constraint: $s^{\alpha}+\frac{4 s}{1-s} \cdot \frac{L(L-3)}{2 \sqrt{\pi}}<1$, when $L>3$, which obviously would put a restriction on $L$ considering $s$ fixed.
} 
which gives (17) provided that $\omega_{0}$ is small enough. We note that choosing $\omega_{0}^{\alpha} \sim L^{-1 / 2}$ allows to choose $c_{L} s^{\alpha} \omega_{0}^{\alpha}$, thus $\sigma$ and $K$ uniformly in $L$. Assume from now on that we fix a choice of $\bar{c}$ and let $\omega_{0}$ such that $\omega_{0}^{\alpha}=\bar{c} L^{-1 / 2}$, $c_{L} s^{\alpha} \omega_{0}^{\alpha}=\delta$ for some fixed $\delta>0$ sufficiently small so that $(1+\delta) s^{\alpha}=: \sigma<1$.

To prove (18), we are going to use that for $f, g \in V_{\alpha}$

$$
\|f g\|_{\alpha} \leq|f|_{\alpha}\|g\|_{\infty}+\|f\|_{1}|g|_{\alpha}+\|f\|_{1}\|g\|_{\infty},
$$

as argued in the proof of Proposition 3.4, [32]. Let $f \in V_{\alpha}$ and $g=1_{X_{\varepsilon}^{0}}$. Recall that $X_{\varepsilon}^{0}=X \backslash H_{\varepsilon}$. Notice that $\operatorname{osc}\left(g, B_{\omega}(x)\right)=1$ if $x \in B_{\omega}\left(\partial X_{\varepsilon}^{0}\right)=B_{\omega}\left(\partial H_{\varepsilon}\right)$. Recall also that $H_{\varepsilon}=\cup_{\mathbf{v} \in V^{+}} \cup_{\mathbf{p} \in \Lambda} H_{\varepsilon}^{\mathbf{p}, \mathbf{v}}$, where the union is not necessarily disjoint. Nevertheless, $\operatorname{osc}\left(g, B_{\omega}(x)\right)$ is either 1 or 0 for $x \in B_{\omega}\left(\partial H_{\varepsilon}^{\mathbf{p}, \mathbf{v}}\right)$ for each $\mathbf{p} \in \Lambda$ and $\mathbf{v} \in V^{+}$, and 0 elsewhere. This implies that

$$
\begin{aligned}
\int_{\mathbb{R}^{L}} \operatorname{Osc}\left(g, B_{\omega}(x)\right) \mathrm{d} x & \leq \sum_{\mathbf{v} \in V^{+}} \sum_{\mathbf{p} \in \Lambda} m_{L}\left(B_{\omega}\left(\partial H_{\varepsilon}^{\mathbf{p}, \mathbf{v}}\right)\right) \\
& \leq L d\left((\varepsilon+2 \omega)^{2}-(\varepsilon-2 \omega)^{2}\right)=8 L d \varepsilon \omega
\end{aligned}
$$

and thus

$$
\sup _{0<\omega \leq \omega_{0}} \omega^{-\alpha} \int_{\mathbb{R}^{L}} \operatorname{osc}\left(g, B_{\omega}(x)\right) \mathrm{d} x \leq 8 L d \varepsilon \omega_{0}^{1-\alpha} .
$$

So $g \in V_{\alpha}$ indeed, and by (20) we obtain

$$
\begin{aligned}
\left\|f 1_{X_{\varepsilon}^{0}}\right\|_{\alpha} & \leq|f|_{\alpha}\left\|1_{X_{\varepsilon}^{0}}\right\|_{\infty}+\|f\|_{1}\left|1_{X_{\varepsilon}^{0}}\right|_{\alpha}+\|f\|_{1}\left\|1_{X_{\varepsilon}^{0}}\right\|_{\infty} \\
& \leq|f|_{\alpha}+\|f\|_{1}+\|f\|_{1}\left|1_{X_{\varepsilon}^{0}}\right|_{\alpha} \\
& \leq\|f\|_{\alpha}+8 L d \varepsilon \omega_{0}^{1-\alpha}\|f\|_{1} .
\end{aligned}
$$

Our choice of $\omega_{0}$ implied that $\omega_{0}^{\alpha} L^{1 / 2} \sim 1$. In turn, $L \omega_{0}^{1-\alpha} \sim L^{\frac{3}{2}-\frac{1}{2 \alpha}}<1$ for sufficiently small $\alpha$. We now fix the value of $\alpha$ such that $\frac{3}{2}-\frac{1}{2 \alpha}<0$, that is, $\alpha \leq \min \{\beta, 1 / 3\}$. Then, $B\left(L, \varepsilon, \omega_{0}\right) \leq 2 \sigma+O(\varepsilon)+K$, in particular

$$
B\left(L, \varepsilon, \omega_{0}\right) \leq C \text { for } \varepsilon \text { sufficiently small. }
$$

Remark 4.3. Note that $f \geq 0$ with $\int_{X} f \mathrm{~d} x=1$, there exists an $n \geq 0$, such that

$$
\left\|\hat{P}_{\varepsilon}^{n} f\right\|_{1}=\int_{X} P_{T_{0}}^{n}\left(1_{X_{\varepsilon}^{n-1}} f\right) \mathrm{d} x=\int_{X} 1_{X_{\varepsilon}^{n-1}} f \mathrm{~d} x<\int_{X} f \mathrm{~d} x=\|f\|_{1} .
$$

Lemma 4.4. Let $C\left(L, \omega_{0}\right)$ be the constant defined by (4). Then,

$$
\sup _{\|f\|_{\alpha} \leq 1}\left\|\left(\hat{P}_{\varepsilon}-P_{T_{0}}\right) f\right\|_{1} \leq C\left(L, \omega_{0}\right) \cdot L \mathrm{~d} \varepsilon^{2} .
$$

Proof. Using (3) we get:

$$
\begin{aligned}
\left\|\left(\hat{P}_{\varepsilon}-P_{T_{0}}\right) f\right\|_{1} & =\left\|P_{T_{0}}\left(1_{X_{\varepsilon}} f\right)-P_{T_{0}} f\right\|_{1} \leq\left\|1_{H_{\varepsilon}} f\right\|_{1} \\
& \leq\left|H_{\varepsilon}\right| \cdot\|f\|_{\infty} \leq C\left(L, \omega_{0}\right)\left|H_{\varepsilon}\right| \cdot\|f\|_{\alpha} .
\end{aligned}
$$

The proof is then completed by noting that $\left|H_{\varepsilon}\right|=\left|\cup_{\mathbf{v} \in V^{+}} \cup_{\mathbf{p} \in \Lambda} H_{\varepsilon}^{\mathbf{p}, \mathbf{v}}\right| \leq$ $L \mathrm{~d} \varepsilon^{2}$. 
Proof of Theorem 2.5. By Lemma 4.2 and the fact that the unit ball of $\left(V_{\alpha}, \|\right.$. $\left.\|_{\alpha}\right)$ is compact in the weak norm $\|\cdot\|_{1}$, the result of [17] implies the residual spectrum of each operator $\hat{P}_{\varepsilon}, \varepsilon \geq 0$ is contained in $\{z \in \mathbb{C}:|z| \leq \sigma\}$. The map $T_{0}$ admits an invariant density $\rho_{0}(x)=\Pi_{i=1}^{L} h\left(x_{i}\right)$. Since $h$ is mixing, $\rho_{0}$ is mixing. This with the quasi-compactness of $P_{0}$ implies $P_{0}$ has a spectral gap when acting on $V_{\alpha}$. By Lemmas 4.2 and 4.4, and the abstract perturbation result of [20] the operator $\hat{P}_{\varepsilon}: V_{\alpha} \rightarrow V_{\alpha}, \varepsilon>0$ has a spectral gap with a leading simple eigenvalue $\lambda_{\varepsilon}$ and by Remark $4.3 \lambda_{\varepsilon} \in(\sigma, 1)$ holds. Moreover, there is $\rho_{\varepsilon} \in V_{\alpha}$, a probability Borel measure $\nu_{\varepsilon}$ and linear operators $Q_{\varepsilon}: V_{\alpha} \rightarrow V_{\alpha}$ such that

with

$$
\lambda_{\varepsilon}^{-1} \hat{P}_{\varepsilon}=\rho_{\varepsilon} \otimes \nu_{\varepsilon}+Q_{\varepsilon}
$$

- $\hat{P}_{\varepsilon} \rho_{\varepsilon}=\lambda_{\varepsilon} \rho_{\varepsilon}, \nu_{\varepsilon} \hat{P}_{\varepsilon}=\lambda_{\varepsilon} \nu_{\varepsilon}, Q_{\varepsilon} \rho_{\varepsilon}=0, \nu_{\varepsilon} Q_{\varepsilon}=0$;

- $\sum_{n=0}^{\infty} \sup _{\varepsilon}\left\|Q_{\varepsilon}^{n}\right\|_{\alpha}<\infty$;

- $\int \rho_{\varepsilon} \mathrm{d} x=1$ and $\sup _{\varepsilon}\left\|\rho_{\varepsilon}\right\|_{\alpha}<\infty$.

We assume the normalization $\nu_{0}\left(\rho_{\varepsilon}\right)=1$ for all $\varepsilon \geq 0$.

We now state some more specific bounds for the above defined quantities. Observing the second item, the spectral radius of $Q_{\varepsilon}$ is some $K_{\varepsilon}(L) \in(0,1)$, so we have

$$
\sum_{k=0}^{\infty}\left\|Q_{\varepsilon}^{k}\right\|_{\alpha} \leq \frac{1}{1-K_{\varepsilon}(L)} \leq \frac{1}{1-K(L)}
$$

for some $K(L)$ that is independent of $\varepsilon$. Moreover, for the last item, since $\sigma$ is a uniform upper bound on the essential spectral radii of $\hat{P}_{\varepsilon}$, we can fix $r \in(\sigma, 1)$ and $\delta_{0} \in(0,1-r)$, both $r$ and $\delta_{0}$ are independent of $L$, so that $B(0, r)$ contains the essential spectrum of $\hat{P}_{\varepsilon}$ and $\lambda_{\varepsilon} \in B\left(1, \delta_{0}\right)$. Then, using the fact that $\rho_{\varepsilon}$ is the nonnegative eigenfunction corresponding to the dominant real eigenvalue $\lambda_{\varepsilon}$, and $\int \rho_{\varepsilon}=1$, by Lemma 4.2 , we have

$$
\lambda_{\varepsilon}\left\|\rho_{\varepsilon}\right\|_{\alpha}=\left\|\hat{P}_{\varepsilon} \rho_{\varepsilon}\right\|_{\alpha} \leq \sigma\left\|\rho_{\varepsilon}\right\|_{\alpha}+B\left(L, \varepsilon, \omega_{0}\right)
$$

Therefore,

$$
\left\|\rho_{\varepsilon}\right\|_{\alpha} \leq \frac{B\left(L, \varepsilon, \omega_{0}\right)}{\lambda_{\varepsilon}-\sigma} \leq \frac{C}{r-\sigma} \quad \text { for } \varepsilon \text { sufficiently small }
$$

by $(21)$.

To obtain the first-order approximation of $\lambda_{\varepsilon}$ we proceed as in [24]. Notice that

$$
\int_{X}\left(\hat{P}_{\varepsilon}-P_{T_{0}}\right) f \mathrm{~d} x=\int_{X} 1_{H_{\varepsilon}} f \mathrm{~d} x
$$

Consequently,

$$
\eta_{\varepsilon}:=\sup _{\|f\|_{\alpha} \leq 1}\left|\int_{X}\left(\hat{P}_{\varepsilon}-P_{T_{0}}\right) f \mathrm{~d} x\right| \leq C\left(L, \omega_{0}\right) \int_{X} 1_{H_{\varepsilon}} \mathrm{d} x \rightarrow 0 \text { as } \varepsilon \rightarrow 0,
$$

where we used that $\|f\|_{\infty} \leq C\left(L, \omega_{0}\right)\|f\|_{\alpha}$ by inequality (3). In particular we obtained that

$$
\eta_{\varepsilon}=O\left(C\left(L, \omega_{0}\right) L \varepsilon^{2}\right)
$$


As $X_{\varepsilon}^{0} \cup H_{\varepsilon}=X$, it holds that $\left|1_{H_{\varepsilon}}\right|_{\alpha}=\left|1_{X_{\varepsilon}^{0}}\right|_{\alpha}$. Thus, we obtain the analogue of (18)

$$
\left\|1_{H_{\varepsilon}} f\right\|_{\alpha} \leq\|f\|_{\alpha}+8 L \mathrm{~d} \varepsilon \omega_{0}^{1-\alpha}\|f\|_{1},
$$

and in particular

$$
\left\|1_{H_{\varepsilon}} \rho_{0}\right\|_{\alpha} \leq\left\|\rho_{0}\right\|_{\alpha}+8 L \mathrm{~d} \varepsilon \omega_{0}^{1-\alpha} .
$$

Using this and (17) we obtain

$$
\begin{aligned}
\left\|P_{T_{0}}\left(1_{H_{\varepsilon}} \rho_{0}\right)\right\|_{\alpha} & \leq \sigma\left\|1_{H_{\varepsilon}} \rho_{0}\right\|_{\alpha}+K \\
& \leq \sigma\left(\left\|\rho_{0}\right\|_{\alpha}+8 L \mathrm{~d} \varepsilon \omega_{0}^{1-\alpha}\right)+K \\
& =\sigma\left\|\rho_{0}\right\|_{\alpha}+B\left(L, \varepsilon, \omega_{0}\right) \\
& \leq \frac{C \sigma}{r-\sigma}+C=\frac{C r}{r-\sigma}=: C_{1}
\end{aligned}
$$

Recall further that $\underline{C}:=\inf _{x} h(x)>0$. Then,

$$
\begin{aligned}
\eta_{\varepsilon} & \cdot\left\|\left(\hat{P}_{\varepsilon}-P_{T_{0}}\right) \rho_{0}\right\|_{\alpha} \\
& \leq C\left(L, \omega_{0}\right) \int_{X} 1_{H_{\varepsilon}} \mathrm{d} x \cdot\left\|P_{T_{0}}\left(1_{H_{\varepsilon}} \rho_{0}\right)\right\|_{\alpha} \leq C_{1} C\left(L, \omega_{0}\right) \int_{X} 1_{H_{\varepsilon}} \mathrm{d} x \\
& \leq \frac{C_{1} C\left(L, \omega_{0}\right)}{\underline{C}} \int_{X} 1_{H_{\varepsilon}} \rho_{0} \mathrm{~d} x=\frac{C_{1} C\left(L, \omega_{0}\right)}{\underline{C}} \cdot \int_{X}\left(\hat{P}_{\varepsilon}-P_{T_{0}}\right) \rho_{0} \mathrm{~d} x
\end{aligned}
$$

Thus, we obtained

$$
\eta_{\varepsilon} \cdot\left\|\left(\hat{P}_{\varepsilon}-P_{T_{0}}\right) \rho_{0}\right\|_{\alpha}=O\left(C\left(L, \omega_{0}\right)\right) \int_{X}\left(\hat{P}_{\varepsilon}-P_{T_{0}}\right) \rho_{0} \mathrm{~d} x .
$$

We now give a bound on $\left|1-\nu_{\varepsilon}\left(\rho_{0}\right)\right|$ following the proof of $[24$, Lemma 6.1 (a)]. We will repeatedly use the fact that $\left(1-\lambda_{\varepsilon}^{-1} \hat{P}_{\varepsilon}\right)\left(\lambda_{\varepsilon}^{-1} \hat{P}_{\varepsilon}\right)^{k}\left(\rho_{0}\right)=(1$ $\left.-\lambda_{\varepsilon}^{-1} \hat{P}_{\varepsilon}\right) Q_{\varepsilon}^{k}\left(\rho_{0}\right)$ for all $k \geq 0$.

Recall the normalization $\nu_{0}\left(\rho_{\varepsilon}\right)=1$ for all $\varepsilon \geq 0$, and that $\lambda_{\varepsilon} \hat{P}_{\varepsilon}$ $=\rho_{\varepsilon} \otimes \nu_{\varepsilon}+Q_{\varepsilon}$. Then, for all $n \in \mathbb{N}$

$$
\nu_{\varepsilon}\left(\rho_{0}\right) \rho_{\varepsilon}=\left(\lambda_{\varepsilon} \hat{P}_{\varepsilon}\right)^{n}\left(\rho_{0}\right)-Q_{\varepsilon}^{n}\left(\rho_{0}\right),
$$

which implies

$$
\nu_{\varepsilon}\left(\rho_{0}\right)=\nu_{0}\left(\left(\lambda_{\varepsilon} \hat{P}_{\varepsilon}\right)^{n}\left(\rho_{0}\right)-Q_{\varepsilon}^{n}\left(\rho_{0}\right)\right) .
$$

Thus, we can write

$$
\begin{aligned}
\left|1-\nu_{\varepsilon}\left(\rho_{0}\right)\right| & =\lim _{n \rightarrow \infty}\left|\nu_{0}\left(\rho_{0}-\left(\lambda_{\varepsilon}^{-1} \hat{P}_{\varepsilon}\right)^{n}\left(\rho_{0}\right)\right)\right| \\
& =\left|\sum_{k=0}^{n-1} \nu_{0}\left(\left[\left(\lambda_{\varepsilon}^{-1} \hat{P}_{\varepsilon}\right)^{k}-\left(\lambda_{\varepsilon}^{-1} \hat{P}_{\varepsilon}\right)^{k+1}\right]\left(\rho_{0}\right)\right)\right| \\
& \leq \sum_{k=0}^{n-1}\left|\nu_{0}\left(\left[\left(1-\lambda_{\varepsilon}^{-1} \hat{P}_{\varepsilon}\right)\left(\lambda_{\varepsilon}^{-1} \hat{P}_{\varepsilon}\right)^{k}\right]\left(\rho_{0}\right)\right)\right| \\
& \leq \sum_{k=0}^{\infty}\left|\nu_{0}\left(\left(1-\lambda_{\varepsilon}^{-1} \hat{P}_{\varepsilon}\right) Q_{\varepsilon}^{k}\left(\rho_{0}\right)\right)\right|
\end{aligned}
$$


as $\left(1-\lambda_{\varepsilon}^{-1} \hat{P}_{\varepsilon}\right)\left(\lambda_{\varepsilon}^{-1} \hat{P}_{\varepsilon}\right)^{k}=\left(1-\lambda_{\varepsilon}^{-1} \hat{P}_{\varepsilon}\right) Q_{\varepsilon}^{k}$. Thus,

$$
\begin{aligned}
\left|1-\nu_{\varepsilon}\left(\rho_{0}\right)\right| & \leq \sum_{k=0}^{\infty}\left|\nu_{0}\left(\left(1-\lambda_{\varepsilon}^{-1} \hat{P}_{\varepsilon}\right) Q_{\varepsilon}^{k}\left(\rho_{0}\right)\right)\right| \\
& \leq \sum_{k=0}^{\infty}\left|\nu_{0}\left(\left(P_{T_{0}}-\hat{P}_{\varepsilon}\right) Q_{\varepsilon}^{k}\left(\rho_{0}\right)\right)\right|+\sum_{k=0}^{\infty}\left|\nu_{0}\left(\left(\hat{P}_{\varepsilon}-\lambda_{\varepsilon}^{-1} \hat{P}_{\varepsilon}\right) Q_{\varepsilon}^{k}\left(\rho_{0}\right)\right)\right| \\
& \leq \sum_{k=0}^{\infty}\left|\nu_{0}\left(\left(P_{T_{0}}-\hat{P}_{\varepsilon}\right) Q_{\varepsilon}^{k}\left(\rho_{0}\right)\right)\right|+\left|\lambda_{\varepsilon}-1\right| \sum_{k=0}^{\infty}\left|\nu_{0}\left(\left(\lambda_{\varepsilon}^{-1} \hat{P}_{\varepsilon}\right) Q_{\varepsilon}^{k}\left(\rho_{0}\right)\right)\right| \\
& =\sum_{k=0}^{\infty}\left|\nu_{0}\left(\left(P_{T_{0}}-\hat{P}_{\varepsilon}\right) Q_{\varepsilon}^{k}\left(\rho_{0}\right)\right)\right|+\left|\lambda_{\varepsilon}-1\right| \sum_{k=0}^{\infty}\left|\nu_{0}\left(Q_{\varepsilon}^{k+1}\left(\rho_{0}\right)\right)\right| \\
& \leq \eta_{\varepsilon} \sum_{k=0}^{\infty}\left\|Q_{\varepsilon}^{k}\right\|_{\alpha} \cdot\left\|\rho_{0}\right\|_{\alpha}+\left|1-\lambda_{\varepsilon}\right|\left\|\nu_{0}\right\|_{\alpha} \sum_{k=1}^{\infty}\left\|Q_{\varepsilon}^{k}\right\|_{\alpha} \cdot\left\|\rho_{0}\right\|_{\alpha} \\
& \leq \frac{C}{1-K(L)}\left(\eta_{\varepsilon}+\left|1-\lambda_{\varepsilon}\right|\right)
\end{aligned}
$$

Since $\left|1-\lambda_{\varepsilon}\right|=\left|\nu_{0}\left(\left(P_{T_{0}}-\hat{P}_{\varepsilon}\right)\left(\rho_{\varepsilon}\right)\right)\right| \leq\left\|\rho_{\varepsilon}\right\|_{\alpha} \eta_{\varepsilon} \leq C \eta_{\varepsilon}$ (by (24)) we obtain that

$$
\left|1-\nu_{\varepsilon}\left(\rho_{0}\right)\right| \leq \frac{C \eta_{\varepsilon}}{1-K(L)} .
$$

Finally, by (23) and (24) we have

$$
\left|1-\nu_{\varepsilon}\left(\rho_{0}\right)\right|=O\left(\frac{C\left(L, \omega_{0}\right) L \varepsilon^{2}}{1-K(L)}\right)
$$

by $(25)$.

In what follows, we bound $\left\|Q_{\varepsilon}^{N}\left(\rho_{0}\right)\right\|_{\alpha}, N \geq 0$ with the help of the tailsum $\kappa_{N}=\sum_{n=N}^{\infty} \sup _{\varepsilon}\left\|Q_{\varepsilon}^{n}\right\|_{\alpha}$. The argument is analogous to [24, Lemma 6.1 (b)].

$$
\begin{aligned}
& \left\|Q_{\varepsilon}^{N}\left(\rho_{0}\right)\right\|_{1} \\
& \quad \leq \varlimsup_{n \rightarrow \infty}\left\|Q_{\varepsilon}^{N}\left(\rho_{0}-\left(\lambda_{\varepsilon}^{-1} \hat{P}_{\varepsilon}\right)^{n}\left(\rho_{0}\right)\right)\right\|_{1}+\overline{\lim }_{n \rightarrow \infty}\left\|Q_{\varepsilon}^{N+n}\left(\rho_{0}\right)\right\|_{\alpha} \\
& \quad \leq \varlimsup_{n \rightarrow \infty}\left\|Q_{\varepsilon}^{N}\left(\sum_{k=0}^{n-1}\left(\lambda_{\varepsilon}^{-1} \hat{P}_{\varepsilon}\right)^{k} \rho_{0}-\left(\lambda_{\varepsilon}^{-1} \hat{P}_{\varepsilon}\right)^{k+1}\left(\rho_{0}\right)\right)\right\|_{1}+\overline{\lim }_{n \rightarrow \infty} \kappa_{N+n}\left\|\rho_{0}\right\|_{\alpha} \\
& \quad \leq \sum_{k=0}^{\infty}\left\|Q_{\varepsilon}^{N}\left(\lambda_{\varepsilon}^{-1} \hat{P}_{\varepsilon}\right)^{k}\left(1-\lambda_{\varepsilon}^{-1} \hat{P}_{\varepsilon}\right)\left(\rho_{0}\right)\right\|_{1}+\overline{\lim }_{n \rightarrow \infty} \kappa_{N+n}\left\|\rho_{0}\right\|_{\alpha} \\
& \quad \leq \sum_{k=0}^{\infty}\left\|Q_{\varepsilon}^{N+k}\left(P_{T_{0}}-\hat{P}_{\varepsilon}+\left(1-\lambda_{\varepsilon}^{-1}\right) \hat{P}_{\varepsilon}\right)\left(\rho_{0}\right)\right\|_{1} \\
& \leq \sum_{k=0}^{\infty}\left(\left\|Q_{\varepsilon}^{N+k}\left(P_{T_{0}}-\hat{P}_{\varepsilon}\right)\left(\rho_{0}\right)\right\|_{1}+\left|1-\lambda_{\varepsilon}^{-1}\right| \cdot\left\|Q_{\varepsilon}^{N+k+1}\left(\rho_{0}\right)\right\|_{1}\right) \\
& \leq \sum_{k=0}^{\infty}\left\|Q_{\varepsilon}^{N+k}\right\|_{\alpha}\left(\left\|\left(P_{T_{0}}-\hat{P}_{\varepsilon}\right)\left(\rho_{0}\right)\right\|_{\alpha}+\left|1-\lambda_{\varepsilon}\right| \cdot\left\|\rho_{0}\right\|_{\alpha}\right),
\end{aligned}
$$


thus

$$
\left\|Q_{\varepsilon}^{N}\left(\rho_{0}\right)\right\|_{1}=O\left(\kappa_{N}\right)\left(\left\|\left(P_{T_{0}}-\hat{P}_{\varepsilon}\right)\left(\rho_{0}\right)\right\|_{\alpha}+\left|1-\lambda_{\varepsilon}\right|\right)
$$

by using (24).

We now prove the formula for $\lambda_{\varepsilon}$ as stated in item 1 of Theorem 2.5. We follow the proof of [24, Theorem 2.1] while tracking the $L$ dependence of the constants. First notice that $\nu_{0}\left(\left(P_{T_{0}}-\hat{P}_{\varepsilon}\right)\left(\rho_{0}\right)\right)=\mu_{0}\left(H_{\varepsilon}\right)$.

$$
\begin{aligned}
\nu_{\varepsilon}\left(\rho_{0}\right)\left(1-\lambda_{\varepsilon}\right) & \\
= & \left(1-\nu_{\varepsilon}\left(\rho_{0}\right)\right) \nu_{0}\left(\left(P_{T_{0}}-\hat{P}_{\varepsilon}\right)\left(\rho_{\varepsilon}\right)\right) \\
= & \mu_{0}\left(H_{\varepsilon}\right)-\nu_{0}\left(\left(P_{T_{0}}-\hat{P}_{\varepsilon}\right)\left(1-\left(\lambda_{\varepsilon}^{-1} \hat{P}_{\varepsilon}\right)^{n}\right)\left(\rho_{0}\right)\right)-\nu_{0}\left(\left(P_{T_{0}}-\hat{P}_{\varepsilon}\right) Q_{\varepsilon}^{n}\left(\rho_{0}\right)\right) \\
= & \mu_{0}\left(H_{\varepsilon}\right)-\sum_{k=0}^{n-1} \nu_{0}\left(\left(P_{T_{0}}-\hat{P}_{\varepsilon}\right)\left(\lambda_{\varepsilon}^{-1} \hat{P}_{\varepsilon}\right)^{k}\left(1-\lambda_{\varepsilon}^{-1} \hat{P}_{\varepsilon}\right)\left(\rho_{0}\right)\right)+O\left(\eta_{\varepsilon}\left\|Q_{\varepsilon}^{n} \rho_{0}\right\|_{1}\right) \\
= & \mu_{0}\left(H_{\varepsilon}\right)-\sum_{k=0}^{n-1} \nu_{0}\left(\left(P_{T_{0}}-\hat{P}_{\varepsilon}\right)\left(\lambda_{\varepsilon}^{-1} \hat{P}_{\varepsilon}\right)^{k}\left(P_{T_{0}}-\hat{P}_{\varepsilon}\right)\left(\rho_{0}\right)\right) \\
& +\left(1-\lambda_{\varepsilon}\right) \sum_{k=0}^{n} \nu_{0}\left(\left(P_{T_{0}}-\hat{P}_{\varepsilon}\right)\left(\lambda_{\varepsilon}^{-1} \hat{P}_{\varepsilon}\right)^{k}\left(\rho_{0}\right)\right) \\
& +O\left(\kappa_{n}\right)\left(O\left(C\left(L, \omega_{0}\right)\right) \mu_{0}\left(H_{\varepsilon}\right)+\eta_{\varepsilon}\left|1-\lambda_{\varepsilon}\right|\right)
\end{aligned}
$$

where for the error term we used (28), then (26). Continuing,

$$
\begin{aligned}
\nu_{\varepsilon}\left(\rho_{0}\right)\left(1-\lambda_{\varepsilon}\right)= & \mu_{0}\left(H_{\varepsilon}\right)\left(1-\sum_{k=0}^{n-1} \lambda_{\varepsilon}^{-k} q_{k, \varepsilon}\right) \\
& +O\left(\eta_{\varepsilon}\right)\left|1-\lambda_{\varepsilon}\right| \sum_{k=0}^{n-1}\left(\left|\nu_{\varepsilon}\left(\rho_{0}\right)\right| \cdot\left\|\rho_{\varepsilon}\right\|_{1}+\left\|Q_{\varepsilon}^{k} \rho_{0}\right\|_{1}\right) \\
& +O\left(\kappa_{n}\right)\left(O\left(C\left(L, \omega_{0}\right)\right) \mu_{0}\left(H_{\varepsilon}\right)+\eta_{\varepsilon}\left|1-\lambda_{\varepsilon}\right|\right)
\end{aligned}
$$

where we used the notation

$$
q_{k, \varepsilon}=\frac{\nu_{0}\left(\left(P_{T_{0}}-\hat{P}_{\varepsilon}\right) \hat{P}_{\varepsilon}^{k}\left(P_{T_{0}}-\hat{P}_{\varepsilon}\right)\left(\rho_{0}\right)\right)}{\nu_{0}\left(\left(P_{T_{0}}-\hat{P}_{\varepsilon}\right)\left(\rho_{0}\right)\right)} .
$$

Using (27), (24) and (23), we can write this as

$$
\begin{aligned}
& \left(1+O\left(\frac{C\left(L, \omega_{0}\right) L \varepsilon^{2}}{1-K(L)}\right)\right)\left(1-\lambda_{\varepsilon}\right)\left(1+n O\left(\eta_{\varepsilon}\right)\right) \\
& =\mu_{0}\left(H_{\varepsilon}\right)\left(1-\sum_{k=0}^{n-1} \lambda_{\varepsilon}^{-k} q_{k, \varepsilon}\right)+O\left(\kappa_{n}\right) C\left(L, \omega_{0}\right) \mu_{0}\left(H_{\varepsilon}\right) \\
& \left(1+O\left(\frac{C\left(L, \omega_{0}\right) L \varepsilon^{2}}{1-K(L)}\right)\right)\left(1-\lambda_{\varepsilon}\right)\left(1+n O\left(C\left(L, \omega_{0}\right) L \varepsilon^{2}\right)\right) \\
& =\mu_{0}\left(H_{\varepsilon}\right)\left(1-\sum_{k=0}^{n-1} \lambda_{\varepsilon}^{-k} q_{k, \varepsilon}\right)+O\left(\kappa_{n}\right) C\left(L, \omega_{0}\right) \mu_{0}\left(H_{\varepsilon}\right)
\end{aligned}
$$


Assuming the $\operatorname{limits}_{\lim _{\varepsilon \rightarrow 0}} q_{k, \varepsilon}=: q_{k}$ exist, this implies that

$$
\lim _{\varepsilon \rightarrow 0} \frac{1-\lambda_{\varepsilon}}{\mu_{0}\left(H_{\varepsilon}\right)}=1-\sum_{k=0}^{n-1} q_{k}+O\left(\kappa_{n}\right),
$$

and finally taking $n \rightarrow \infty$ gives the formula

$$
\lim _{\varepsilon \rightarrow 0} \frac{1-\lambda_{\varepsilon}}{\mu_{0}\left(H_{\varepsilon}\right)}=\theta
$$

by using the notation $\theta=1-\sum_{k=0}^{\infty} q_{k}$.

We now compute the values of $q_{k}, k \geq 0$ to obtain an explicit formula for $\theta$. To this end, we recall from [19] that $q_{k, \varepsilon}$ can be computed as

$$
\begin{aligned}
q_{k, \varepsilon} & =\frac{1}{\mu_{0}\left(H_{\varepsilon}\right)} \int_{H_{\varepsilon}} \hat{P}_{\varepsilon}^{k} P_{T_{0}}\left(1_{H_{\varepsilon}} \rho_{0}\right) \mathrm{d} x \\
& =\frac{\mu_{0}\left(H_{\varepsilon} \cap T_{0}^{-1} H_{\varepsilon}^{c} \cap \cdots T_{0}^{-k} H_{\varepsilon}^{c} \cap T_{0}^{-(k+1)} H_{\varepsilon}\right)}{\mu_{0}\left(H_{\varepsilon}\right)}
\end{aligned}
$$

Recall further that $H_{\varepsilon}=\cup_{\mathbf{v} \in V^{+}} \cup_{\mathbf{p} \in \Lambda} H_{\varepsilon}^{\mathbf{p}, \mathbf{v}}$ where

$$
H_{\varepsilon}^{\mathbf{p}, \mathbf{v}}=\left\{x_{\mathbf{p}} \in A_{\varepsilon, \mathbf{v}}, x_{\mathbf{p}+\mathbf{v}} \in A_{\varepsilon,-\mathbf{v}}, x_{\mathbf{q}} \in I \text { for } \mathbf{q} \neq \mathbf{p}, \mathbf{p}+\mathbf{v}\right\},
$$

and $I_{\mathbf{p}, \mathbf{v}}=\lim _{\varepsilon \rightarrow 0} H_{\varepsilon}^{\mathbf{p}, \mathbf{v}}$, i.e.,

$$
I_{\mathbf{p}, \mathbf{v}}=\left\{x_{\mathbf{p}}=a_{\mathbf{v}}, x_{\mathbf{p}+\mathbf{v}}=a_{-\mathbf{v}}, x_{\mathbf{q}} \in I \text { for } \mathbf{q} \neq \mathbf{p}, \mathbf{p}+\mathbf{v}\right\},
$$

where $a_{\mathbf{v}}=\lim _{\varepsilon \rightarrow 0} A_{\varepsilon, \mathbf{v}}$ and $a_{-\mathbf{v}}=\lim _{\varepsilon \rightarrow 0} A_{\varepsilon,-\mathbf{v}}$.

Consider first the case when none of the elements of $S=\left\{\left(a_{\mathbf{v}}, a_{-\mathbf{v}}\right)\right\}_{\mathbf{v} \in V^{+}}$ are periodic points, that is, $S^{p e r}=\emptyset$. Then, by the expression (31) it is evident that in the $\varepsilon \rightarrow 0$ limit we get $q_{k}=0$ for all $k \geq 0$. As a consequence, $\theta=1$.

For each $\left(a_{\mathbf{v}}, a_{-\mathbf{v}}\right) \in S^{\text {per }}$ recall that $k(\mathbf{v})$ is the smallest integer such that $T_{2,0}^{k+1}\left(a_{\mathbf{v}}, a_{-\mathbf{v}}\right) \in S$, and $V_{k}^{+} \subset V^{+}$is the set of directions for which $k(\mathbf{v})=k$ and $K=\{k(\mathbf{v})\}_{\mathbf{v} \in V^{+}}$. Notice that $S$ can be divided into periodic orbits, where the first preimage of each point intersecting $S$ is well-defined. We will denote the said preimage of $\left(a_{\mathbf{v}}, a_{-\mathbf{v}}\right)$ by $\left(\tau_{*}^{-\left(k^{\prime}+1\right)}\left(a_{\mathbf{v}}\right), \tau_{*}^{-\left(k^{\prime}+1\right)}\left(a_{-\mathbf{v}}\right)\right)$. Consider now some $k \in K$. Computing $q_{k, \varepsilon}$, we get

$$
\begin{aligned}
q_{k, \varepsilon} & =\frac{1}{\mu_{0}\left(H_{\varepsilon}\right)} \int_{H_{\varepsilon}} P_{\varepsilon}^{k} P_{T_{0}}\left(1_{H_{\varepsilon}} \rho_{0}\right) \mathrm{d} x \\
& =\frac{1}{\mu_{0}\left(H_{\varepsilon}\right)} \int_{H_{\varepsilon}^{k}} \sum_{y \in T_{0}^{-(k+1)}(x)} \frac{\rho_{0}(y)}{\left\|D T_{0}^{k+1} y\right\|} \mathrm{d} x \\
& =\frac{1}{\mu_{0}\left(H_{\varepsilon}\right)} \int_{H_{\varepsilon}^{k}} \sum_{y \in T_{0}^{-(k+1)}(x)} \frac{\prod_{\mathbf{p} \in \Lambda} h\left(y_{\mathbf{p}}\right)}{\prod_{\mathbf{p} \in \Lambda}\left|\left(\tau^{k+1}\right)^{\prime}\left(y_{\mathbf{p}}\right)\right|} \mathrm{d} x
\end{aligned}
$$

where $H_{\varepsilon}^{k}=H_{\varepsilon} \cap T_{0}\left(H_{\varepsilon}^{c}\right) \cap \cdots \cap T_{0}^{k}\left(H_{\varepsilon}^{c}\right) \cap T_{0}^{k+1}\left(H_{\varepsilon}\right)$.

As a notational ease write

$$
F(x)=\sum_{y \in T_{0}^{-(k+1)}(x)} \frac{\prod_{\mathbf{p} \in \Lambda} h\left(y_{\mathbf{p}}\right)}{\prod_{\mathbf{p} \in \Lambda}\left|\left(\tau^{k+1}\right)^{\prime}\left(y_{\mathbf{p}}\right)\right|} .
$$


We remark that $F=P_{T_{0}^{k+1}} \rho_{0}=\rho_{0}$, but we prefer to keep this expression for reasons that will become clear in what follows.

Recall that we defined $\Lambda^{\ell}=\left\{\left(\mathbf{p}_{1}, \ldots, \mathbf{p}_{k}\right): \mathbf{p}_{i} \in \Lambda, i=1, \ldots, \ell\right\},\left(V^{+}\right)^{\ell}$ $=\left\{\left(\mathbf{v}_{1}, \ldots, \mathbf{v}_{\ell}\right): \mathbf{v}_{i} \in V^{+}, i=1, \ldots, \ell\right\}$ and let $\left(V_{k}^{+}\right)^{\ell}=\left\{\left(\mathbf{v}_{1}, \ldots, \mathbf{v}_{\ell}\right): \mathbf{v}_{i} \in\right.$ $\left.V_{k}^{+}, i=1, \ldots, \ell\right\}$. Recalling (10) we can write

$$
\int_{H_{\varepsilon}^{k}} F(x) \mathrm{d} x=\sum_{\ell=1}^{\lfloor L / 2\rfloor}(-1)^{\ell+1} \sum_{\substack{\left\{\mathbf{p}_{i}\right\}_{i=1}^{\ell} \in \Lambda^{\ell}\left\{\mathbf{v}_{i}\right\}_{i=1}^{\ell} \in\left(V^{+}\right)^{\ell} \\ \mathbf{p}_{i}<\mathbf{p}_{i+1}}} \sum_{\left(\cap_{i=1}^{\ell} H_{\varepsilon}^{\mathbf{p}_{i}, \mathbf{v}_{i}}\right) \cap H_{\varepsilon}^{k}} F(x) \mathrm{d} x .
$$

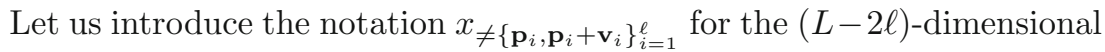
vector that we get from $\left\{x_{\mathbf{q}}\right\}_{\mathbf{q} \in \Lambda}$ by discarding the coordinates $x_{\left\{\mathbf{p}_{i}, \mathbf{p}_{i}+\mathbf{v}_{i}\right\}_{i=1}^{\ell}}$. Assume $\left\{\mathbf{v}_{i}\right\}_{i=1}^{\ell} \in\left(V_{k}^{+}\right)^{\ell}$. Then, $\left(\cap_{i=1}^{\ell} H_{\varepsilon}^{\mathbf{p}_{i}, \mathbf{v}_{i}}\right) \cap H_{\varepsilon}^{k}=\cap_{i=1}^{\ell} H_{\varepsilon}^{\mathbf{p}_{i}, \mathbf{v}_{i}}$ for small enough $\varepsilon$. We now compute the limit

$$
\lim _{\varepsilon \rightarrow 0} \frac{\mu_{0}\left(\cap_{i=1}^{\ell} H_{\varepsilon}^{\mathbf{p}_{i}, \mathbf{v}_{i}}\right)}{\mu_{0}\left(H_{\varepsilon}\right)} \cdot \frac{1}{\mu_{0}\left(\cap_{i=1}^{\ell} H_{\varepsilon}^{\mathbf{p}_{i}, \mathbf{v}_{i}}\right)} \int_{\cap_{i=1}^{\ell} H_{\varepsilon}^{\mathbf{p}_{i}, \mathbf{v}_{i}}} F(x) \mathrm{d} x .
$$

Since $F$ is continuous at $x \in S$ (based on the continuity assumptions and $\tau$ and $\tau^{\prime}$, implying the continuity of $h$ ), we obtain

$$
\begin{aligned}
\lim _{\varepsilon \rightarrow 0} & \frac{1}{\mu_{0}\left(\cap_{i=1}^{\ell} H_{\varepsilon}^{\mathbf{p}_{i}, \mathbf{v}_{i}}\right)} \int_{\cap_{i=1}^{\ell} H_{\varepsilon}^{\mathbf{p}_{i}, \mathbf{v}_{i}}} F(x) \mathrm{d} x \\
= & \prod_{i=1}^{\ell} \frac{h\left(\tau_{*}^{-(k+1)}\left(a_{\mathbf{v}_{i}}\right)\right) h\left(\tau_{*}^{-(k+1)}\left(a_{-\mathbf{v}_{i}}\right)\right)}{\left|\left(\tau^{k+1}\right)^{\prime}\left(\tau_{*}^{-(k+1)}\left(a_{\mathbf{v}_{i}}\right)\right)\left(\tau^{k+1}\right)^{\prime}\left(\tau_{*}^{-(k+1)}\left(a_{-\mathbf{v}_{i}}\right)\right)\right|} \cdot \frac{1}{h\left(a_{\mathbf{v}_{i}}\right) h\left(a_{-\mathbf{v}_{i}}\right)} \\
& \times \int_{I^{L-2 \ell}} \sum_{y \in T_{0}^{-(k+1)}(x)} \prod_{\mathbf{q} \notin\left\{\mathbf{p}_{i}, \mathbf{p}_{i}+\mathbf{v}_{i}\right\}_{i=1}^{\ell}} \frac{h\left(y_{\mathbf{q}}\right)}{\left|\left(\tau^{k+1}\right)^{\prime}\left(y_{\mathbf{q}}\right)\right|} \mathrm{d} x_{\neq\left\{\mathbf{p}_{i}, \mathbf{p}_{i}+\mathbf{v}_{i}\right\}_{i=1}^{\ell}} \\
& \times \chi\left(\cap_{i=1}^{\ell} I_{\mathbf{p}_{i}, \mathbf{v}_{i}}\right)
\end{aligned}
$$

where $\chi(A)=1$ if $A$ is non-empty and $\chi(A)=0$ otherwise. Notice that

$$
\int_{I^{L-2 \ell}} \sum_{y \in T_{0}^{-(k+1)}(x)} \prod_{\substack{\mathbf{q} \in \Lambda \\ \mathbf{q} \notin\left\{\mathbf{p}_{i}, \mathbf{p}_{i}+\mathbf{v}_{i}\right\}_{i=1}^{\ell}}} \frac{h\left(y_{\mathbf{q}}\right)}{\left|\left(\tau^{k+1}\right)^{\prime}\left(y_{\mathbf{q}}\right)\right|} \mathrm{d} x_{\neq\left\{\mathbf{p}_{i}, \mathbf{p}_{i}+\mathbf{v}_{i}\right\}_{i=1}^{\ell}}=1
$$

by integrating successively according to each variable $x_{\mathbf{q}}$ and using that $P_{\tau} h$ $=h$ and $\int_{I} h=1$. Then, we can write

$$
\begin{aligned}
\lim _{\varepsilon \rightarrow 0} & \frac{1}{\mu_{0}\left(\cap_{i=1}^{\ell} H_{\varepsilon}^{\mathbf{p}_{i}, \mathbf{v}_{i}}\right)} \int_{\cap_{i=1}^{\ell} H_{\varepsilon}^{\mathbf{p}_{i}, \mathbf{v}_{i}}} F(x) \mathrm{d} x \\
= & \prod_{i=1}^{\ell} \frac{h\left(\tau_{*}^{-(k+1)}\left(a_{\mathbf{v}_{i}}\right)\right) h\left(\tau_{*}^{-(k+1)}\left(a_{-\mathbf{v}_{i}}\right)\right)}{\left|\left(\tau^{k+1}\right)^{\prime}\left(\tau_{*}^{-(k+1)}\left(a_{\mathbf{v}_{i}}\right)\right)\left(\tau^{k+1}\right)^{\prime}\left(\tau_{*}^{-(k+1)}\left(a_{-\mathbf{v}_{i}}\right)\right)\right|} \cdot \frac{1}{h\left(a_{\mathbf{v}_{i}}\right) h\left(a_{-\mathbf{v}_{i}}\right)} \\
& \times \chi\left(\cap_{i=1}^{\ell} I_{\mathbf{p}_{i}, \mathbf{v}_{i}}\right) .
\end{aligned}
$$

Notice that $\left(\tau_{*}^{-(k+1)}\left(a_{\mathbf{v}_{i}}\right), \tau_{*}^{-(k+1)}\left(a_{-\mathbf{v}_{i}}\right)\right)=\left(a_{\mathbf{v}_{j}}, a_{-\mathbf{v}_{j}}\right)$ for some $\mathbf{v}_{j} \in V_{k}^{+}$. 
On the other hand, using (9) we obtain

$$
\begin{aligned}
& \lim _{\varepsilon \rightarrow 0} \frac{\mu_{0}\left(\cap_{i=1}^{\ell} H_{\varepsilon}^{\mathbf{p}_{i}, \mathbf{v}_{i}}\right)}{\mu_{0}\left(H_{\varepsilon}\right)} \\
& =\lim _{\varepsilon \rightarrow 0} \frac{\mu_{0}\left(\cap_{i=1}^{\ell} H_{\varepsilon}^{\mathbf{p}_{i}, \mathbf{v}_{i}}\right)}{\sum_{k=1}^{L / 2\rfloor}(-1)^{k+1} \sum_{\substack{\left\{\mathbf{p}_{i}\right\}_{i=1}^{k} \in \Lambda^{k} \\
\mathbf{p}_{i}<\mathbf{p}_{i+1}}} \sum_{\left\{\mathbf{v}_{i}\right\}_{i=1}^{k} \in\left(V^{+}\right)^{k}} \mu_{0}\left(\cap_{i=1}^{k} H_{\varepsilon}^{\mathbf{p}_{i}, \mathbf{v}_{i}}\right)} \\
& \quad= \begin{cases}\frac{h\left(a_{\mathbf{v}_{1}}\right) h\left(a_{-\mathbf{v}_{1}}\right)}{\sum_{\mathbf{p} \in \Lambda} \sum_{\mathbf{v} \in V^{+}} h\left(a_{\mathbf{v}}\right) h\left(a_{-\mathbf{v}}\right)} & \text { if } \ell=1, \\
0 & \text { otherwise. }\end{cases}
\end{aligned}
$$

The reason we get zero in the limit for $\ell>1$ is that $m_{L-2}\left(\cap_{i=1}^{\ell} I_{\varepsilon}^{\mathbf{p}_{i}, \mathbf{v}_{i}}\right)=0$ while $m_{L-2}\left(I_{\varepsilon}^{\mathbf{p}_{i}, \mathbf{v}_{i}}\right)>0$ (we have $m_{L-2}\left(I_{\varepsilon}^{\mathbf{p}_{i}, \mathbf{v}_{i}}\right)=1$ in fact.) This implies

$$
\begin{aligned}
& \lim _{\varepsilon \rightarrow 0} \frac{\mu_{0}\left(\cap_{i=1}^{\ell} H_{\varepsilon}^{\mathbf{p}_{i}, \mathbf{v}_{i}}\right) / \varepsilon^{2}}{\sum_{k=1}^{L / 2\rfloor}(-1)^{k+1} \sum_{\substack{\left\{\mathbf{p}_{i}\right\}_{i=1}^{k} \in \Lambda^{k} \\
\mathbf{p}_{i}<\mathbf{p}_{i+1}}} \sum_{\left\{\mathbf{v}_{i}\right\}_{i=1}^{k} \in\left(V^{+}\right)^{k}} \mu_{0}\left(\bigcap_{i=1}^{k} H_{\varepsilon}^{\mathbf{p}_{i}, \mathbf{v}_{i}}\right) / \varepsilon^{2}} \\
& =\lim _{\varepsilon \rightarrow 0} \frac{\mu_{0}\left(\cap_{i=1}^{\ell} H_{\varepsilon}^{\mathbf{p}_{i}, \mathbf{v}_{i}}\right) / \varepsilon^{2^{\ell}} \cdot \varepsilon^{2(\ell-1)}}{\sum_{k=1}^{L / 2\rfloor}(-1)^{k+1} \sum_{\substack{\left\{\mathbf{p}_{i}\right\}_{i=1}^{k} \in \Lambda^{k} \\
\mathbf{p}_{i}<\mathbf{p}_{i+1}}} \sum_{\left\{\mathbf{v}_{i}\right\}_{i=1}^{k} \in\left(V^{+}\right)^{k}} \mu_{0}\left(\bigcap_{i=1}^{k} H_{\varepsilon}^{\mathbf{p}_{i}, \mathbf{v}_{i}}\right) / \varepsilon^{2^{k}} \cdot \varepsilon^{2(k-1)}} \\
& =\lim _{\varepsilon \rightarrow 0} \frac{\prod_{i=1}^{\ell} h\left(a_{\mathbf{v}_{i}}\right) h\left(a_{-\mathbf{v}_{i}}\right) \varepsilon^{2(\ell-1)}}{\sum_{k=1}^{\lfloor L / 2\rfloor}(-1)^{k+1} \sum_{\substack{\left\{\mathbf{p}_{i}\right\}_{i=1}^{k} \in \Lambda^{k} \\
\mathbf{p}_{i}<\mathbf{p}_{i+1}}} \sum_{\left\{\mathbf{v}_{i}\right\}_{i=1}^{k} \in\left(V^{+}\right)^{k}} \prod_{i=1}^{k} h\left(a_{\mathbf{v}_{i}}\right) h\left(a_{-\mathbf{v}_{i}}\right) \varepsilon^{2(k-1)}} \\
& =\frac{0}{\sum_{\mathbf{p} \in \Lambda} \sum_{\mathbf{v} \in V^{+}} h\left(a_{\mathbf{v}}\right) h\left(a_{-\mathbf{v}}\right)+0}=0 .
\end{aligned}
$$

To finish the proof, consider (32), keep only the term $\ell=1$ and repeating this computation for each $\mathbf{p} \in \Lambda$ and $\mathbf{v} \in V_{k}^{+}$, we obtain

$$
\begin{aligned}
q_{k} & =\frac{1}{\sum_{\mathbf{p} \in \Lambda} \sum_{\mathbf{v} \in V^{+}} h\left(a_{\mathbf{v}}\right) h\left(a_{-\mathbf{v}}\right)} \sum_{\mathbf{p} \in \Lambda} \sum_{\mathbf{v} \in V_{k}^{+}} \frac{h\left(a_{\mathbf{v}}\right) h\left(a_{-\mathbf{v}}\right)}{\left|\left(\tau^{k+1}\right)^{\prime}\left(a_{\mathbf{v}}\right)\left(\tau^{k+1}\right)^{\prime}\left(a_{-\mathbf{v}}\right)\right|} \\
& =\frac{1}{\sum_{\mathbf{v} \in V^{+}} h\left(a_{\mathbf{v}}\right) h\left(a_{-\mathbf{v}}\right)} \sum_{\mathbf{v} \in V_{k}^{+}} \frac{h\left(a_{\mathbf{v}}\right) h\left(a_{-\mathbf{v}}\right)}{\left|\left(\tau^{k+1}\right)^{\prime}\left(a_{\mathbf{v}}\right)\left(\tau^{k+1}\right)^{\prime}\left(a_{-\mathbf{v}}\right)\right|} .
\end{aligned}
$$

Finally,

$$
\begin{aligned}
\theta & =1-\sum_{k \in K} q_{k} \\
& =1-\frac{1}{\sum_{\mathbf{v} \in V^{+}} h\left(a_{\mathbf{v}}\right) h\left(a_{-\mathbf{v}}\right)} \sum_{k \in K} \sum_{\mathbf{v} \in V_{k}^{+}} \frac{h\left(a_{\mathbf{v}}\right) h\left(a_{-\mathbf{v}}\right)}{\left|\left(\tau^{k+1}\right)^{\prime}\left(a_{\mathbf{v}}\right)\left(\tau^{k+1}\right)^{\prime}\left(a_{-\mathbf{v}}\right)\right|} .
\end{aligned}
$$


Lemma 4.1 readily implies that $\mu_{0}\left(H_{\varepsilon}\right)=L \Xi_{\varepsilon}(1+o(1)) \sim \varepsilon^{2} L d$ as

$$
d \underline{C}^{2} \varepsilon^{2} \leq \sum_{\mathbf{v} \in V^{+}} \int_{A_{\varepsilon, \mathbf{v}}} \int_{A_{\varepsilon,-\mathbf{v}}} h\left(x_{1}\right) h\left(x_{2}\right) \mathrm{d} x_{1} \mathrm{~d} x_{2} \leq d \bar{C}^{2} \varepsilon^{2}
$$

where $\bar{C}, \underline{C}$ are the respective upper and lower bounds on $h$ noted previously. The $o(1)$ error term is understood in the limit $L \varepsilon^{2} \rightarrow 0$, which for fixed $L$ is equivalent to $\varepsilon \rightarrow 0$.

To prove the second item, first notice that

$$
\begin{aligned}
m_{L}\left(X_{\varepsilon}^{n-1}\right) & =\int_{X} 1_{X_{\varepsilon}^{n-1}}(x) \mathrm{d} x=\int_{X} P_{T_{0}}^{n}\left(1_{X_{\varepsilon}^{n-1}}(x)\right) \mathrm{d} x=\int_{X} \hat{P}_{\varepsilon}^{n} 1(x) \mathrm{d} x \\
& =\lambda_{\varepsilon}^{n}\left[\int_{X} \rho_{\varepsilon} \otimes \nu_{\varepsilon}(1) \mathrm{d} x+\int_{X} Q_{\varepsilon}^{n} 1(x) \mathrm{d} x\right],
\end{aligned}
$$

where in the last step we have used the spectral decomposition (22). Consequently,

$$
\frac{1}{n} \ln \left(m_{L}\left(X_{\varepsilon}^{n-1}\right)\right)-\ln \lambda_{\varepsilon}=\frac{1}{n} \ln \left[\int_{X} \rho_{\varepsilon} \otimes \nu_{\varepsilon}(1) \mathrm{d} x+\int_{X} Q_{\varepsilon}^{n} 1(x) \mathrm{d} x\right] .
$$

Since $\left\|Q_{\varepsilon}\right\|_{1} \leq\left\|Q_{\varepsilon}\right\|_{\alpha}<1, \exists C>0$ such that

$$
\left|\frac{1}{n} \ln \left(m_{L}\left(X_{\varepsilon}^{n-1}\right)\right)-\ln \lambda_{\varepsilon}\right| \leq \frac{C}{n} .
$$

Hence,

$$
\lim _{n \rightarrow \infty} \frac{1}{n} \ln \left(m_{L}\left(X_{\varepsilon}^{n-1}\right)\right)=\ln \lambda_{\varepsilon}
$$

and the first collision rate, with respect to $m_{L}$ in the $L$-dimensional system is given by $\hat{r}_{L}=-\ln \lambda_{\varepsilon}$. Using the asymptotics of $\lambda_{\varepsilon}$, we have

$$
\begin{aligned}
\lambda_{\varepsilon} & =1-\mu_{0}\left(H_{\varepsilon}\right) \cdot \theta(1+o(1)) \\
& =e^{-\mu_{0}\left(H_{\varepsilon}\right) \cdot \theta(1+o(1))}
\end{aligned}
$$

This implies

$$
\hat{r}_{L}=\mu_{0}\left(H_{\varepsilon}\right) \cdot \theta(1+o(1)) .
$$

The proof of the third item of the theorem follows from item 1 along the lines of Proposition 2 of [19].

We choose $N$ such that $N \eta_{\varepsilon}+O\left(C\left(L, \omega_{0}\right) \kappa_{N}\right)$ is minimal. Denote

$$
\zeta(L)=|\log (1-K(L))|
$$

where $K(L)$ was defined by (23). Thus, $N=O\left(\frac{1}{\zeta(L)} \cdot\left|\log \left(\frac{\eta_{\varepsilon}}{C\left(L, \omega_{0}\right) \zeta(L)}\right)\right|\right)$. Set $\delta_{\varepsilon}=O\left(\frac{\eta_{\varepsilon}}{\zeta(L)}\left|\log \frac{\eta_{\varepsilon}}{C\left(L, \omega_{0}\right) \zeta(L)}\right|\right)$.

Let $\xi_{\varepsilon}=\theta_{N, \varepsilon}+O\left(C\left(L, \omega_{0}\right) \kappa_{N}\right)$ where $\theta_{N, \varepsilon}=\sum_{k=0}^{N-1} \lambda_{\varepsilon}^{-k} q_{k, \varepsilon}$. By (29) we obtain that

$$
\begin{aligned}
\lambda_{\varepsilon} & =1-\mu_{0}\left(H_{\varepsilon}\right) \xi_{\varepsilon}\left(1+O\left(N \eta_{\varepsilon}\right)\right) \\
& =\exp \left[-\mu_{0}\left(H_{\varepsilon}\right) \xi_{\varepsilon}\left(1+O\left(\delta_{\varepsilon}\right)\right)\right]
\end{aligned}
$$


As $\lambda_{\varepsilon}=1-\mu_{0}\left(H_{\varepsilon}\right)(\theta+o(1))$ by (30) we also have $\lim _{\varepsilon \rightarrow 0} \xi_{\varepsilon}=\theta>0$, in particular $\xi_{\varepsilon}>0$ for $\varepsilon$ small enough.

Let $n=\left\lfloor t /\left(\xi_{\varepsilon} \mu_{0}\left(H_{\varepsilon}\right)\right)\right\rfloor$. First assume that $n>\left|\frac{\log \eta_{\varepsilon}}{\zeta(L)}\right|$. Then, as argued in $[19$, Equation (26)], we can write

$$
\begin{aligned}
\mu_{0}\left\{t_{\varepsilon} \geq \frac{t}{\xi_{\varepsilon} \mu_{0}\left(H_{\varepsilon}\right)}\right\} & =\lambda_{\varepsilon}^{n+q}\left(\nu_{\varepsilon}\left(\rho_{0}\right)+O\left(\left\|Q_{\varepsilon}^{n}\right\|_{\alpha}\right)\right) \\
& =\exp \left[-t\left(1+O\left(\delta_{\varepsilon}\right)\right)\right] \cdot\left(1+O\left(\frac{C\left(L, \omega_{0}\right) L \varepsilon^{2}}{1-K(L)}\right)\right)
\end{aligned}
$$

where in the last line we used (27). So for $\varepsilon$ small enough we have

$$
\begin{aligned}
\left|\mu_{0}\left\{t_{\varepsilon} \geq \frac{t}{\xi_{\varepsilon} \mu_{0}\left(H_{\varepsilon}\right)}\right\}-e^{-t}\right| & \leq C(t \vee 1) e^{-t} \delta_{\varepsilon} \\
& \leq C(t \vee 1) e^{-t} \frac{\eta_{\varepsilon}}{\zeta(L)} \log \frac{\eta_{\varepsilon}}{C\left(L, \omega_{0}\right) \zeta(L)} \\
& \leq C(t \vee 1) e^{-t} C\left(L, \omega_{0}\right) \frac{L \varepsilon^{2}}{\zeta(L)} \log \frac{L \varepsilon^{2}}{\zeta(L)}
\end{aligned}
$$

The case of $n \leq\left|\frac{\log \eta_{\varepsilon}}{\zeta(L)}\right|$ can be argued in the same way as in the proof of [19, Proposition 2].

Proof of Theorem 2.8. To derive the expression in the theorem, we consider

$$
\frac{\hat{r}_{L}}{\mu_{0}\left(H_{\varepsilon}\right)} \cdot \frac{\mu_{0}\left(H_{\varepsilon}\right)}{L \Xi_{\varepsilon}}
$$

as $\varepsilon \rightarrow 0$. By equation $(7)$

$$
\frac{\hat{r}_{L}}{\mu_{0}\left(H_{\varepsilon}\right)}=\theta(1+o(1))
$$

in the limit as $\varepsilon \rightarrow 0$. Moreover, equation (8) implies

$$
\frac{\mu_{0}\left(H_{\varepsilon}\right)}{L \Xi_{\varepsilon}}=1+o(1)
$$

as $\varepsilon \rightarrow 0$. This completes the proof.

We finish the paper by a remark on considering the infinite-dimensional limit of the collision rate and its per lattice unit counterpart.

Remark 4.5. If we want to study collision rates in the infinite limit of the system, i.e., as $L \rightarrow \infty$, we need an asymptotic formula for $\lambda_{\varepsilon}$ in some kind of joint limit where $L \rightarrow \infty$ and $\varepsilon \rightarrow 0$. To this end, recall (29) :

$$
\begin{aligned}
\left(1+O\left(\frac{C\left(L, \omega_{0}\right) L \varepsilon^{2}}{1-K(L)}\right)\right) & \frac{1-\lambda_{\varepsilon}}{\mu_{0}\left(H_{\varepsilon}\right)}\left(1+n O\left(C\left(L, \omega_{0}\right) L \varepsilon^{2}\right)\right) \\
& =1-\sum_{k=0}^{n-1} \lambda_{\varepsilon}^{-k} q_{k, \varepsilon}+O\left(\kappa_{n}\right) C\left(L, \omega_{0}\right)
\end{aligned}
$$

The term $O\left(\kappa_{n}\right) C\left(L, \omega_{0}\right)$ causes serious difficulties in taking any kind of limit in which $L \rightarrow \infty$, as $C\left(L, \omega_{0}\right)$, defined by (4) diverges as $L \rightarrow \infty$. We note that 
the constant $C\left(L, \omega_{0}\right)$ appears in that term due to $(26)$, the analogue of (A6) in [24]. In addition, the term $K(L)$ (a bound on the modulus of the second largest eigenvalue of $\hat{P}_{\varepsilon}$ ) is also difficult to control.

\section{Acknowledgements}

We would like to thank Gerhard Keller for his comments on an earlier version of this work, which were crucial for the content and the presentation of the paper. We further thank Péter Bálint for inspiring discussions and an anonymous referee for helpful comments.

Open Access. This article is licensed under a Creative Commons Attribution 4.0 International License, which permits use, sharing, adaptation, distribution and reproduction in any medium or format, as long as you give appropriate credit to the original author(s) and the source, provide a link to the Creative Commons licence, and indicate if changes were made. The images or other third party material in this article are included in the article's Creative Commons licence, unless indicated otherwise in a credit line to the material. If material is not included in the article's Creative Commons licence and your intended use is not permitted by statutory regulation or exceeds the permitted use, you will need to obtain permission directly from the copyright holder. To view a copy of this licence, visit http://creativecommons. org/licenses/by/4.0/.

Publisher's Note Springer Nature remains neutral with regard to jurisdictional claims in published maps and institutional affiliations.

\section{References}

[1] Abadi, M.: Sharp error terms and neccessary conditions for exponential hitting times in mixing processes. Ann. Probab. 32(1A), 243-264 (2004)

[2] Bahsoun, W., Liverani, C.: Anosov diffeomorphisms, anisotropic BV spaces and regularity of foliations. Ergodic Theory Dyn. Syst. 1-37 (2021)

[3] Bálint, P., Gilbert, T., Nándori, P., Szász, D., Tóth, I.-P.: On the limiting Markov process of energy exchanges in a rarely interacting ball-piston gas. J. Stat. Phys. 166(3-4), 903-925 (2017)

[4] Bardet, J.-B., Fernandez, B.: Extensive escape rate in lattices of weakly coupled expanding maps. Discrete Contin. Dyn. Syst. 31(3), 669-684 (2011)

[5] Bernardin, C., Huveneers, F., Lebowitz, J.L., Liverani, C., Olla, S.: Green-Kubo formula for weakly coupled systems with noise. Commun. Math. Phys. 334(3), 1377-1412 (2015)

[6] Boyarsky, A., Góra, P.: Laws of Chaos. Birkhäuser: Boston (1997)

[7] Bruin, H., Demers, M.F., Todd, M.: Hitting and escaping statistics: mixing, targets and holes. Adv. Math. 328, 1263-1298 (2018)

[8] Bunimovich, L.A., Sinai, Y.G.: Spacetime chaos in coupled map lattices. Nonlinearity 1, 491-516 (1988)

[9] Bunimovich, L.A., Yurchenko, A.: Where to place a hole to achieve a maximal escape rate. Israel. J. Math. 182, 229-252 (2011) 
[10] Chazottes, J.R., Fernandez, B. (eds.): Dynamics of Coupled Map Lattices and of Related Spatially Extended Systems, vol. 671. Springer, Berlin (2005)

[11] Collet, P., Eckmann, J.-P.: The definition and measurement of the topological entropy per unit volume in parabolic PDEs. Nonlinearity 12(3), 451-473 (1999)

[12] Coutinho, R., Fernandez, B.: Extensive bounds on the topological entropy of repellers in piecewise expanding coupled map lattices. Ergodic Theory Dyn. Syst. 33(3), 870-895 (2013)

[13] Demers, M.F., Young, L.-S.: Escape rates and conditionally invariant measures. Nonlinearity 19(2), 377-397 (2006)

[14] Fernandez, B.: Breaking of ergodicity in expanding systems of globally coupled piecewise affine circle maps. J. Stat. Phys. 154, 999-1029 (2014)

[15] Gaspard, P., Gilbert, T.: Heat conduction and Fourier's law by consecutive local mixing and thermalization. Phys. Rev. Lett. 101(2), 20601 (2008)

[16] Góra, P., Boyarsky, A.: Absolutely continuous invariant measures for piecewise expanding $C^{2}$ transformation in $R^{N}$. Israel J. Math. 67(3), 272-286 (1989)

[17] Hennion, H.: Sur un théorème spectral et son application aux noyaux Lipchitziens. Proc. Am. Math. Soc. 118, 627-634 (1993)

[18] Hirata, M., Saussol, M., Vaienti, S.: Statistics of return times: a general framework and new applications. Commun. Math. Phys. 206(1), 33-55 (1999)

[19] Keller, G.: Rare events, exponential hitting times and extremal indices via spectral perturbation. Dyn. Syst. 27(1), 11-27 (2012)

[20] Keller, G., Liverani, C.: Stability of the spectrum for transfer operators. Ann. Scuola Norm. Sup. Pisa Cl. Sci. 28(1), 141-152 (1999)

[21] Keller, G., Liverani, C.: A spectral gap for a one-dimensional lattice of coupled piecewise expanding interval maps. In: Dynamics of Coupled Map Lattices and of Related Spatially Extended Systems, pp. 115-151. Springer, Berlin (2005)

[22] Keller, G., Liverani, C.: Uniqueness of the SRB measure for piecewise expanding weakly coupled map lattices in any dimension. Commun. Math. Phys. 262(1), $33-50$ (2006)

[23] Keller, G., Liverani, C.: Map lattices coupled by collisions. Commun. Math. Phys. 291(2), 591-597 (2009)

[24] Keller, G., Liverani, C.: Rare events, escape rates and quasistationarity: some exact formulae. J. Stat. Phys. 135(3), 519-534 (2009)

[25] Keller, G., Künzle, M., Nowicki, T.: Some phase transitions in coupled map lattices. Physica D Nonlinear Phenom. 59, 39-51 (1992)

[26] Kaneko, K.: Overview of coupled map lattices. Chaos 2, 279-282 (1992)

[27] Koiller, J., Young, L.S.: Coupled map networks. Nonlinearity 23, 1121-1141 (2010)

[28] Liverani, C.: Multidimensional expanding maps with singularities: a pedestrian approach. Ergodic Theory Dyn. Syst. 33(1), 168-182 (2013)

[29] Pereira, T., van Strien, S., Tanzi, M.: Heterogeneously coupled maps: hub dynamics and emergence across connectivity layers. J. Eur. Math. Soc. (JEMS) 22, 2183-2252 (2020)

[30] Pollicott, M., Urbański, M.: Open Conformal Systems and Perturbations of Transfer Operators. Lecture Notes in Mathematics, vol. 2206. Springer, Cham (2017) 
[31] Ruelle, D.: A mechanical model for Fourier's law of heat conduction. Commun. Math. Phys. 311(3), 755-768 (2012)

[32] Saussol, B.: Absolutely continuous invariant measures for multidimensional expanding maps. Israel J. Math. 116, 223-248 (2000)

[33] Sélley, F.M.: Asymptotic properties of mean field coupled maps. Ph.D. thesis, Budapest University of Technology and Economics (2019)

[34] Young, L.-S.: Understanding chaotic dynamical systems. Commun. Pure Appl. Math. 66(9), 1439-1463 (2013)

Wael Bahsoun

Department of Mathematical Sciences

Loughborough University

Loughborough, Leicestershire LE11 3TU

UK

e-mail: W.Bahsoun@lboro.ac.uk

Fanni M. Sélley

Mathematical Institute of Leiden University

Niels Bohrweg 1

2333 CA Leiden

The Netherlands

e-mail: f.m.selley@math.leidenuniv.nl

Communicated by Dmitry Dolgopyat.

Received: August 13, 2021.

Accepted: January 26, 2022. 